\title{
Spectral probes of the holographic Fermi groundstate: dialing between the electron star and AdS Dirac hair
}

\author{
Mihailo Čubrović, Yan Liu, Koenraad Schalm, Ya-Wen Sun, and Jan Zaanen \\ Institute Lorentz for Theoretical Physics, Leiden University \\ P.O. Box 9506, Leiden 2300RA, The Netherlands*
}

\begin{abstract}
We argue that the electron star and the AdS Dirac hair solution are two limits of the free charged Fermi gas in AdS. Spectral functions of holographic duals to probe fermions in the background of electron stars have a free parameter that quantifies the number of constituent fermions that make up the charge and energy density characterizing the electron star solution. The strict electron star limit takes this number to be infinite. The Dirac hair solution is the limit where this number is unity. This is evident in the behavior of the distribution of holographically dual Fermi surfaces. As we decrease the number of constituents in a fixed electron star background the number of Fermi surfaces also decreases. An improved holographic Fermi groundstate should be a configuration that shares the qualitative properties of both limits.
\end{abstract}

\footnotetext{
*Email addresses: cubrovic, liu, kschalm, sun, jan@lorentz.leidenuniv.nl.
} 


\section{INTRODUCTION}

The insight provided by the application of the AdS/CFT correspondence to finite density Fermi systems has given brand new perspectives on the theoretical robustness of non-Fermi liquids [1-3]; on an understanding of the non-perturbative stability of the regular Fermi liquid equivalent to order parameter universality for bosons [4, 5], and most importantly on the notion of fermionic criticality: Fermi systems with no scale. In essence strongly coupled conformally invariant fermi systems are one answer to the grand theoretical question of fermionic condensed matter: Are there finite density Fermi systems that do not refer at any stage to an underlying perturbative Fermi gas?

It is natural to ask to what extent AdS/CFT can provide a more complete answer to this question. Assuming, almost tautologically, that the underlying system is strongly coupled and there is in addition some notion of a large $N$ limit, the Fermi system is dual to classical general relativity with a negative cosmological constant coupled to charged fermions and electromagnetism. As AdS/CFT maps quantum numbers to quantum numbers, finite density configurations of the strongly coupled large $N$ system correspond to solutions of this Einstein-Maxwell-Dirac theory with finite charge density. Since the AdS fermions are the only object carrying charge, and the gravity system is weakly coupled, one is immediately inclined to infer that the generic solution is a weakly coupled charged Fermi gas coupled to AdS gravity: in other words an AdS electron star [6, 7], the charged equivalent of a neutron star in asymptotically anti-de Sitter space [8, 9].

Nothing can seem more straightforward. Given the total charge density $Q$ of interest, one constructs the free fermionic wavefunctions in this system, and fills them one by one in increasing energy until the total charge equals $Q$. For macroscopic values of $Q$ these fermions themselves will backreact on the geometry. One can compute this backreaction; it changes the potential for the free fermions at subleading order. Correcting the wavefunctions at this subleading order, one converges on the true solution order by order in the gravitational strength $\kappa^{2} E_{\text {full system. }}^{2}$. Here $E_{\text {full system }}$ is the energy carried by the Fermi system and $\kappa^{2}$ is the gravitational coupling constant $\kappa^{2}=8 \pi G_{\text {Newton }}$ in the AdS gravity system. Perturbation theory in $\kappa$ is dual to the $1 / N$ expansion in the associated condensed matter system.

The starting point of the backreaction computation is to follow Tolman-OppenheimerVolkov (TOV) and use a Thomas-Fermi (TF) approximation for the lowest order one-loop 
contribution [6 9]. The Thomas-Fermi approximation applies when the number of constituent fermions making up the Fermi gas is infinite. For neutral fermions this equates to the statement that the energy-spacing between the levels is neglible compared to the chemical potential associated with $Q, \Delta E / \mu \rightarrow 0$. For charged fermions the Thomas-Fermi limit is more direct: it is the limit $q / Q \rightarrow 0$ where $q$ is the charge of each constituent fermion. ${ }^{1}$

This has been the guiding principle behind the approaches [6-11] and the recent papers [12, 13, with the natural assumption that all corrections beyond Thomas-Fermi are small quantitative changes rather than qualitative ones. On closer inspection, however, this completely natural TF-electron star poses a number of puzzles. The most prominent perhaps arises from the AdS/CFT correspondence finding that every normalizable fermionic wavefunction in the gravitational bulk corresponds to a fermionic quasiparticle excitation in the dual condensed matter system. In particular occupying a particular wavefunction is dual to having a particular Fermi-liquid state [4]. In the Thomas Fermi limit the gravity dual thus describes an infinity of Fermi liquids, whereas the generic condensed matter expectation would have been that a been that a single(/few) liquid(s) would be the generic groundstate away from the strongly coupled fermionic quantum critical point at zero charge density. This zoo of Fermi surfaces is already present in the grand canonical approaches at fixed $\mu$ (extremal AdS-Reissner-Nordström (AdS-RN) black holes) [3] and a natural explanation would be that this is a large $N$ effect. This idea, that the gravity theory is dual to a condensed matter system with $N$ species of fermions, and increasing the charge density "populates" more and more of the distinct species of Fermi liquids, is very surprising from the condensed matter perspective. Away from criticality one would expect the generic groundstate to be a single Fermi-liquid or some broken state due to pairing. To pose the puzzle sharply, once one has a fermionic quasiparticle one should be able to adiabatically continue it to a free Fermi gas, which would imply that the free limit of the strongly coupled fermionic CFT is not a single but a system of order $N$ fermions with an ordered distribution of fermi-momenta. A possible explanation of the multitude of Fermi surfaces that is consistent with a single Fermi surface at weak coupling is that AdS/CFT describes so-called "deconfined and/or fractionalized Fermi-liquids" where the number of Fermi surfaces is directly tied to the coupling

\footnotetext{
${ }^{1}$ For a fermion in an harmonic oscillator potential $E_{n}=\hbar(n-1 / 2) \omega$ : thus $\Delta E / E_{\text {total }}=1 / \sum_{1}^{N}(n-1 / 2)=$ $2 / N^{2}$.
} 
strength [12 16]. It would argue that fermionic quantum criticality goes hand in hand with fractionalization for which there is currently scant experimental evidence.

The second puzzle is more technical. Since quantum numbers in the gravity system equal the quantum numbers in the dual condensed matter system, one is inclined to infer that each subsequent AdS fermion wavefunction has incrementally higher energy than the previous one. Yet analyticity of the Dirac equation implies that all normalizable wavefunctions must have strictly vanishing energy [17]. It poses the question how the order in which the fermions populate the Fermi gas is determined.

The third puzzle is that in the Thomas Fermi limit the Fermi gas is gravitationally strictly confined to a bounded region: famously, the TOV-neutron star has an edge. In AdS/CFT, however, all information about the dual condensed matter system is read off at asymptotic AdS infinity. Qualitatively, one can think of AdS/CFT as an "experiment" analogous to probing a spatially confined Fermi gas with a tunneling microscope held to the exterior of the trap. Extracting the information of the dual condensed matter system is probing the AdS Dirac system confined by a gravitoelectric trap instead of a magneto-optical trap for cold atoms. Although the Thomas-Fermi limit should reliably capture the charge and energy densities in the system, its abrupt non-analytic change at the edge (in a trapped system) and effective absence of a density far away from the center are well known to cause qualitative deficiencies in the description of the system. Specifically Friedel oscillations - quantum interference in the outside tails of the charged fermion density, controlled by the ratio $q / Q$ and measured by a tunneling microscope — are absent. Analogously, there could be qualitative features in the AdS asymptotics of both the gravito-electric background and the Dirac wavefunctions in that adjusted background that are missed by the TF-approximation. The AdS asymptotics in turn specify the physics of the dual condensed matter system and since our main interest is to use AdS/CFT to understand quantum critical fermion systems where $q / Q$ is finite, the possibility of a qualitative change inherent in the Thomas Fermi limit should be considered.

There is another candidate AdS description of the dual of a strongly coupled finite density Fermi system: the AdS black hole with Dirac hair [4, 5]. One arrives at this solution when one starts one's reasoning from the dual condensed matter system, rather than the Dirac fields in AdS gravity. Insisting that the system collapses to a generic single species Fermi-liquid ground state, the dual gravity description is that of an AdS Einstein-Dirac-Maxwell system 
with a single nonzero normalizable Dirac wavefunction. To have a macroscopic backreaction the charge of this single Dirac field must be macroscopic. The intuitive way to view this solution is as the other simplest approximation to free Fermi gas coupled to gravity. What we mean is that the full gravito-electric response is in all cases controlled by the total charge $Q$ of the solution: as charge is conserved it is proportional to the constituent charge $q$ times the number of fermions $n_{F_{A d S}}$ and the two simple limits correspond to $n_{F} \rightarrow \infty, q \rightarrow 0$ with $Q=q n_{F}$ fixed or $n_{F} \rightarrow 1, q \rightarrow Q$. The former is the Thomas-Fermi electron star, the latter is the AdS Dirac hair solution. In the context of AdS/CFT there is a significant difference between the two solutions in that the Dirac Hair solution clearly does not give rise to the puzzles 1, 2 and 3: there is by construction no zoo of Fermi-surfaces and therefore no ordering. Moreover since the wavefunction is demanded to be normalizable, it manifestly encodes the properties of the system at the AdS boundary. On the other hand the AdS Dirac hair solution does pose the puzzle that under normal conditions the total charge $Q$ is much larger than the constituent charge $q$ both from the gravity/string theory point of view and the condensed matter perspective. Generically one would expect a Fermi gas electron star rather than Dirac hair.

In this article we shall provide evidence for this point of view that the AdS electron star and the AdS Dirac hair solution are two limits of the same underlying system. Specifically we shall show that (1) the electron star solution indeed has the constituent charge as a free parameter which is formally sent to zero to obtain the Thomas-Fermi approximation. (2) The number of normalizable wavefunctions in the electron star depend on the value of the constituent charge $q$. We show this by computing the electron star spectral functions. They depend in similar way on $q$ as the first AdS/CFT Fermi system studies in an AdS$\mathrm{RN}$ background. In the formal limit where $q \rightarrow Q$, only one normalizable mode remains and the spectral function wavefunction resembles the Dirac Hair solution, underlining their underlying equivalence. Since both approximations have qualitative differences as a description of the AdS dual to strongly coupled fermionic systems, it argues that an improved approximation which has characteristics of both is called for.

The results here are complimentary to and share an analysis of electron star spectral functions with the two recent articles [12] and [13] that appeared in the course of this work (see also [18] for fermion spectral functions in general Lifshitz backgrounds). Our motivation to probe the system away from the direct electron star limit differs: we have therefore been 
more precise in defining this limit and in the analysis of the Dirac equation in the electron star background.

\section{EINSTEIN-MAXWELL THEORY COUPLED TO CHARGED FERMIONS}

The Lagrangian that describes both the electron star and Dirac Hair approximation is Einstein-Maxwell theory coupled to charged matter

$$
S=\int d^{4} x \sqrt{-g}\left[\frac{1}{2 \kappa^{2}}\left(R+\frac{6}{L^{2}}\right)-\frac{1}{4 q^{2}} F^{2}+\mathcal{L}_{\text {matter }}\left(e_{\mu}^{A}, A_{\mu}\right)\right]
$$

where $L$ is the AdS radius, $q$ is the electric charge and $\kappa$ is the gravitational coupling constant. It is useful to scale the electromagnetic interaction to be of the same order as the gravitational interaction and measure all lengths in terms of the AdS radius $L$ :

$$
g_{\mu \nu} \rightarrow L^{2} g_{\mu \nu}, \quad A_{\mu} \rightarrow \frac{q L}{\kappa} A_{\mu}
$$

The system then becomes

$$
S=\int d^{4} x \sqrt{-g}\left[\frac{L^{2}}{2 \kappa^{2}}\left(R+6-\frac{1}{2} F^{2}\right)+L^{4} \mathcal{L}_{\text {matter }}\left(L e_{\mu}^{A}, \frac{q L}{\kappa} A_{\mu}\right)\right] .
$$

Note that in the rescaled variables the effective charge of charged matter now depends on the ratio of the electromagnetic to gravitational coupling constant: $q_{\text {eff }}=q L / \kappa$. For the case of interest, charged fermions, the Lagrangian in these variables is

$$
L^{4} \mathcal{L}_{\text {fermions }}\left(L e_{\mu}^{A}, \frac{q L}{\kappa} A_{\mu}\right)=-\frac{L^{2}}{\kappa^{2}} \bar{\Psi}\left[e_{A}^{\mu} \Gamma^{A}\left(\partial_{\mu}+\frac{1}{4} \omega_{\mu}^{B C} \Gamma_{B C}-i \frac{q L}{\kappa} A_{\mu}\right)-m L\right] \Psi,
$$

where $\bar{\Psi}$ is defined as $\bar{\Psi}=i \Psi^{\dagger} \Gamma^{0}$. Compared to the conventional normalization the Dirac

field has been made dimensionless $\Psi=\kappa \sqrt{L} \psi_{\text {conventional }}$. With this normalization all terms in the action have a factor $L^{2} / \kappa^{2}$ and it will therefore scale out of the equations of motion

$$
\begin{aligned}
R_{\mu \nu}-\frac{1}{2} g_{\mu \nu} R-3 g_{\mu \nu} & =\left(F_{\mu \rho} F_{\nu}^{\rho}-\frac{1}{4} g_{\mu \nu} F_{\rho \sigma} F^{\rho \sigma}+T_{\mu \nu}^{\text {fermions }}\right), \\
D_{\mu} F^{\mu \nu} & =-q_{\text {eff }} J_{\text {fermions }}^{\nu}
\end{aligned}
$$

with

$$
\begin{aligned}
T_{\mu \nu}^{\text {fermions }}= & \frac{1}{2} \bar{\Psi} e_{A(\mu} \Gamma^{A}\left[\partial_{\nu)}+\frac{1}{4} \omega_{\nu)}^{B C} \Gamma_{B C}-i \frac{q L}{\kappa} A_{\nu)}\right] \Psi-\frac{\kappa^{2} L^{2}}{2} g_{\mu \nu} \mathcal{L}_{\text {fermions }} \\
J_{\text {fermions }}^{\nu} & =i \bar{\Psi} e_{A}^{\nu} \Gamma^{A} \Psi
\end{aligned}
$$


where the symmetrization is defined as $B_{(\mu} C_{\nu)}=B_{\mu} C_{\nu}+B_{\nu} C_{\mu}$ and the Dirac equation

$$
\left[e_{A}^{\mu} \Gamma^{A}\left(\partial_{\mu}+\frac{1}{4} \omega_{\mu}^{B C} \Gamma_{B C}-i \frac{q L}{\kappa} A_{\mu}\right)-m L\right] \Psi=0 .
$$

The stress-tensor and current are to be evaluated in the specific state of the system. For a single excited wavefunction, obeying (2.8), this gives the AdS Dirac hair solution constructed in [4]. (More specifically, the Dirac hair solution consists of a radially isotropic set of wavefunctions with identical momentum size $|\vec{k}|=\sqrt{k_{x}^{2}+k_{y}^{2}}$, such that the Pauli principle plays no role.) For multiple occupied fermion states, even without backreaction due to gravity, adding the contributions of each separate solution to (2.8) rapidly becomes very involved. In such a many-body-system, the collective effect of the multiple occupied fermion states is better captured in a "fluid" approximation

$$
T_{\mu \nu}^{\text {fluid }}=(\rho+p) u_{\mu} u_{\nu}+p g_{\mu \nu}, \quad N_{\mu}^{\text {fluid }}=n u_{\mu}
$$

with

$$
\rho=\left\langle u^{\mu} T_{\mu \nu} u^{\nu}\right\rangle_{\text {matter only }}, \quad n=-\left\langle u_{\mu} J^{\mu}\right\rangle_{\text {matter only }}
$$

In the center-of-mass rest frame of the multiple fermion system $\left(u_{\mu}=\left(e_{t \underline{0}}, 0,0,0\right)\right)$, the expressions for the stress-tensor and charge density are given by the one-loop equal-time expectation values (as opposed to time-ordered correlation functions)

$$
\rho=\left\langle\bar{\Psi}(t) e_{\underline{0}}^{t} \Gamma^{\underline{0}}\left(\partial_{t}+\frac{1}{4} \omega_{t}^{A B} \Gamma_{A B}-i q_{\mathrm{eff}} A_{t}\right) \Psi(t)\right\rangle .
$$

By the optical theorem the expectation value is equal to twice imaginary part of the Feynman propagator $^{2}$

$$
\rho=\lim _{t \rightarrow t^{\prime}} 2 \operatorname{Im} \operatorname{Tr}\left[e_{\underline{0}}^{t} \Gamma^{\underline{0}}\left(\partial_{t}+\frac{1}{4} \omega_{t}^{A B} \Gamma_{A B}-i q_{\mathrm{eff}} A_{t}\right) G_{F}^{A d S}\left(t^{\prime}, t\right)\right] .
$$

In all situations of interest, all background fields will only have dependence on the radial AdS direction; in that case the spin connection can be absorbed in the normalization of the spinor wavefunction. ${ }^{3}$ In an adiabatic approximation for the radial dependence of $e_{t \underline{0}}$ and $A_{t}$

\footnotetext{
${ }^{2}$ From unitarity for the $S$ matrix $S^{\dagger} S=1$ one obtains the optical theorem $T^{\dagger} T=2 \operatorname{Im} T$ for the transition matrix $T$ defined as $S \equiv 1+i T$.

3 i.e. one can redefine spinors $\chi(r)=f(r) \Psi(r)$ such that the connection term is no longer present in the equation of motion.
} 
- where $\mu_{\text {loc }}(r)=q_{\text {eff }} e_{\underline{0}}^{t}(r) A_{t}(r)$ and $\omega(r)=-i e_{\underline{0}}^{t}(r) \partial_{t} ;$ - this yields the known expression for a many-body-fermion system at finite chemical potential

$$
\begin{aligned}
\rho(r) & =\lim _{\beta \rightarrow \infty} 2 \int \frac{d^{3} k d \omega}{(2 \pi)^{4}}\left[\omega(r)-\mu_{\text {loc }}(r)\right] \operatorname{Im} \operatorname{Tr} i \Gamma^{\underline{0}} G_{F}^{\beta}(\omega, k) \\
& =\lim _{\beta \rightarrow \infty} \int \frac{d k d \omega}{4 \pi^{3}}\left[k^{2}(\omega-\mu)\right]\left[\frac{1}{2}-\frac{1}{2} \tanh \left(\frac{\beta}{2}(\omega-\mu)\right)\right] \operatorname{Tr}\left(i \Gamma^{0}\right)^{2} \frac{\kappa^{2}}{L^{2}} \pi \delta\left((\omega-\mu)-\sqrt{k^{2}+(m L)^{2}}\right) \\
& =\lim _{\beta \rightarrow \infty} \frac{\kappa^{2}}{\pi^{2} L^{2}} \int d \omega f_{F D}(\beta(\omega-\mu))\left[(\omega-\mu)^{2}-(m L)^{2}\right][\omega-\mu] \frac{(\omega-\mu) \theta(\omega-\mu-m L)}{\sqrt{(\omega-\mu)^{2}-(m L)^{2}}} \\
& =\frac{1}{\pi^{2}} \frac{\kappa^{2}}{L^{2}} \int_{m L}^{\mu_{\mathrm{loc}}} d E E^{2} \sqrt{E^{2}-(m L)^{2}}
\end{aligned}
$$

The normalization $\kappa^{2} / L^{2}$ follows from the unconventional normalization of the Dirac field in eq. 2.4. ${ }^{4}$ Similarly

$$
n=\frac{1}{\pi^{2}} \frac{\kappa^{2}}{L^{2}} \int_{m L}^{\mu_{\mathrm{loc}}} d E E \sqrt{E^{2}-(m L)^{2}}=\frac{1}{3 \pi^{2}} \frac{\kappa^{2}}{L^{2}}\left(\mu_{\mathrm{loc}}{ }^{2}-(m L)^{2}\right)^{3 / 2} .
$$

The adiabatic approximation is valid for highly localized wavefunctions, i.e. the expression must be dominated by high momenta (especially in the radial direction). The exact expression on the other hand will not have a continuum of solutions to the harmonic condition $-\Gamma^{0} \omega+\Gamma^{i} k_{i}+\Gamma^{z} k_{z}-\Gamma^{0} \mu_{\mathrm{loc}}-i m L=0$. Normalizable solutions to the AdS Dirac equations only occur at discrete momenta - one can think of the gravitational background as a potential well. The adiabatic approximation is therefore equivalent to the Thomas-Fermi approximation for a Fermi-gas in a box.

To get an estimate for the parameter range where the adiabatic approximation holds, consider the adiabatic bound $\partial_{r} \mu_{\text {loc }}(r) \ll \mu_{\text {loc }}(r)^{2}$. Using the field equation for $A_{\underline{0}}=$ $\mu_{\text {loc }} / q_{\text {eff }}:$

$$
\partial_{r}^{2} \mu_{\mathrm{loc}} \sim q_{\mathrm{eff}}^{2} n
$$

this bound is equivalent to requiring

$$
\partial_{r}^{2} \mu_{\text {loc }} \ll \partial_{r} \mu_{1 \mathrm{oc}}^{2} \Rightarrow\left(\frac{q L}{\kappa}\right)^{2} n \ll 2 \mu_{\text {loc }} \partial_{r} \mu_{\text {loc }} \quad \Rightarrow \quad\left(\frac{q L}{\kappa}\right)^{2} n \ll \mu_{\text {loc }}^{3}
$$

${ }^{4}$ One can see this readily by converting the dimensionless definition of $\rho$, eq 2.11 , to the standard dimension. Using capitals for dimensionless quantities and lower-case for dimensionful ones

$$
\rho \sim\left\langle\Psi \partial_{T} \Psi\right\rangle \sim \kappa^{2} L^{2}\left\langle\psi \partial_{t} \psi\right\rangle \sim \kappa^{2} L^{2} \int_{m}^{\mu} d \epsilon \epsilon^{2} \sqrt{\epsilon^{2}-m^{2}} \sim \frac{\kappa^{2}}{L^{2}} \int_{m L}^{\mu L} d E E^{2} \sqrt{E^{2}-(m L)^{2}}
$$

with $\mu L=\mu_{\text {loc }}$ above. 
where in the last line we used the original bound again. If the chemical potential scale is considerably higher than the mass of the fermion, we may use 2.14 to approximate $n \sim \frac{\kappa^{2}}{L^{2}} \mu_{1 \mathrm{oc}}^{3}$. Thus the adiabatic bound is equivalent to,

$$
q=\frac{q_{\text {eff }} \kappa}{L} \ll 1
$$

the statement that the constituent charge of the fermions is infinitesimal. Note that in the rescaled action 2.3, 2.4,$L / \kappa$ plays the role of $1 / \hbar$, and eq. 2.17) is thus equivalent to the semiclassical limit $\hbar \rightarrow 0$ with $q_{\text {eff }}$ fixed. Since AdS/CFT relates $L / \kappa \sim N_{c}$ this acquires the meaning in the context of holography that there is a large $N_{c}$ scaling limit [12, 13] of the CFT with fermionic operators where the RG-flow is "adiabatic". Returning to the gravitational description the additional assumption that the chemical potential is much larger than the mass is equivalent to

$$
\begin{aligned}
\frac{Q_{\text {phys }}^{\text {total }}}{V_{\text {spatial AdS }}}=\frac{L Q_{\text {eff }}^{\text {total }}}{\kappa V_{\text {spatial AdS }}} & \equiv \frac{L}{\kappa V_{\text {spatial AdS }}} \int d r \sqrt{-g_{\text {induced }}}\left(q_{\text {eff }} n\right) \\
& \simeq \frac{1}{V_{\text {spatial AdS }}} \int d r \sqrt{-g} \frac{q_{\text {eff }} \kappa}{L} \mu_{\text {loc }}^{3}(r) \gg q(m L)^{3} .
\end{aligned}
$$

This implies that the total charge density in AdS is much larger than that of a single charged particle (as long as $m L \sim 1$ ). The adiabatic limit is therefore equivalent to a thermodynamic limit where the Fermi gas consists of an infinite number of constituents, $n \rightarrow \infty, q \rightarrow 0$ such that the total charge $Q \sim n q$ remains finite.

The adiabatic limit of a many-body fermion system coupled to gravity are the TolmanOppenheimer-Volkov equations. Solving this in asymptotically AdS gives us the charged neutron or electron star constructed in [7]. Knowing the quantitative form of the adiabatic limit, it is now easy to distinguish the electron star solution from the "single wavefunction" Dirac Hair solution. The latter is trivially the single particle limit $n \rightarrow 1, q \rightarrow Q$ with the total charge $Q$ finite. The electron star and Dirac Hair black hole are opposing limitsolutions of the same system. We shall now make this connection more visible by identifying a formal dialing parameter that interpolates between the two solutions.

To do so we shall need the full adiabatic Tolman-Oppenheimer-Volkov equations for the AdS electron star [7]. Since the fluid is homogeneous and isotropic, the background metric and electrostatic potential will respect these symmetries and will be of the form (recall that we are already using "dimensionless" lengths, eq. (2.2)

$$
d s^{2}=-f(r) d t^{2}+g(r) d r^{2}+r^{2}\left(d x^{2}+d y^{2}\right), \quad A=h(r) d t,
$$


where $f(r), g(r), h(r)$ are functions of $r$; the horizon is located at $r=0$ and the boundary is at $r=\infty$. Combining this ansatz with a rescaling $m L=q_{\text {eff }} \hat{m}$ the bosonic background equations of motion become [7]

$$
\begin{aligned}
\frac{1}{r}\left(\frac{f^{\prime}}{f}+\frac{g^{\prime}}{g}\right)-\frac{g h \sigma}{\sqrt{f}} & =0, & \rho & =\frac{q_{\mathrm{eff}}^{4} \kappa^{2}}{\pi^{2} L^{2}} \int_{\hat{m}}^{\frac{h}{\sqrt{f}}} d \epsilon \epsilon^{2} \sqrt{\epsilon^{2}-\hat{m}^{2}}, \\
\frac{f^{\prime}}{r f}+\frac{h^{\prime 2}}{2 f}-g(3+p)+\frac{1}{r^{2}} & =0, & \sigma & =\frac{q_{\mathrm{eff}}^{4} \kappa^{2}}{\pi^{2} L^{2}} \int_{\hat{m}}^{\frac{h}{\sqrt{f}}} d \epsilon \epsilon \sqrt{\epsilon^{2}-\hat{m}^{2}}, \\
h^{\prime \prime}+\frac{2}{r} h^{\prime}-\frac{g \sigma}{\sqrt{f}}\left(\frac{r h h^{\prime}}{2}+f\right) & =0, & & -p=\rho-\frac{h}{\sqrt{f}} \sigma,
\end{aligned}
$$

where we have used that $\mu_{1 \mathrm{oc}}=q_{\mathrm{eff}} h / \sqrt{f}$ and $\sigma=n q_{\mathrm{eff}}$ is the rescaled local charge density. What one immediately notes is that the Tolman-Oppenheimer-Volkov equations of motion for the background only depend on the parameters $\hat{\beta} \equiv \frac{q_{\text {eff }}^{4} \kappa^{2}}{\pi^{2} L^{2}}$ and $\hat{m}$, whereas the original Lagrangian and the fermion equation of motion also depend on $q_{\mathrm{eff}}=\left(\frac{\pi^{2} L^{2} \hat{\beta}}{\kappa^{2}}\right)^{1 / 4}$. It is therefore natural to guess that the parameter $q_{\text {eff }}=q L / \kappa$ will be the interpolating parameter away from the adiabatic electron star limit towards the Dirac Hair BH.

Indeed in these natural electron star variables the adiabatic bound (2.17) translates into

$$
\hat{\beta} \ll \frac{L^{2}}{\kappa^{2}}=\frac{q_{\text {eff }}^{2}}{q^{2}} .
$$

Thus we see that for a given electron star background with $\hat{\beta}$ fixed decreasing $\kappa / L$ improves the adiabatic fluid approximation whereas increasing $\kappa / L$ makes the adiabatic approximation poorer and poorer. "Dialing $\kappa / L \mathrm{up} /$ down" therefore interpolates between the electron star and the Dirac Hair BH. Counterintuively improving adiabaticity by decreasing $\kappa / L$ corresponds to increasing $q_{\text {eff }}$ for fixed $q$, but this is just a consequence of recasting the system in natural electron star variables. A better way to view improving adiabaticity is to decrease the microscopic charge $q$ but while keeping $q_{\text {eff }}$ fixed; this shows that a better way to think of $q_{\text {eff }}$ is as the total charge rather than the efffective constituent charge.

The parameter $\kappa / L=q / q_{\text {eff }}$ parametrizes the gravitational coupling strength in units of the AdS curvature, and one might worry that "dialing $\kappa / L$ up" pushes one outside the regime of classical gravity. This is not the case. One can easily have $\hat{\beta} \gg 1$ and tune $\kappa / L$ towards or away from the adiabatic limit within the regime of classical gravity. From eq. 2.17) we see that the edge of validity of the adiabatic regime $\hat{\beta} \simeq L^{2} / \kappa^{2}$ is simply equivalent to a microscopic charge $q=1$ which clearly has a classical gravity description. It is not hard to see that the statement above is the equivalent of changing the level splitting in the Fermi 
gas, while keeping the overall energy/charge fixed. In a Fermi gas microscopically both the overall energy and the level splitting depends on $\hbar$. Naively increasing $\hbar$ increases both, but one can move away from the adiabatic limit either by decreasing the overall charge density, keeping $\hbar$ fixed or by keeping the charge density fixed and raising $\hbar$. Using again the analogy between $\kappa / L$ and $\hbar$, the electron star situation is qualitatively the same where one should think of $\hat{\beta} \sim q^{4} L^{2} / \kappa^{2}$ parametrizing the microscopic charge. One can either insist on keeping $\kappa / L$ fixed and increase the microscopic charge $\hat{\beta}$ to increase the level splitting or one can keep $\hat{\beta}$ fixed and increase $\kappa / L$. In the electron star, however, the background geometry changes with $\hat{\beta}$ in addition to the level splitting, and it is therefore more straightforward to keep $\hat{\beta}$ and the geometry fixed, while dialing $\kappa / L$.

We will now give evidence for our claim that the electron star and Dirac Hair solution are two opposing limits. To do so, we need to identify an observable that goes either beyond the adiabatic background approximation or beyond the single particle approximation. Since the generic intermediate state is still a many-body fermion system, the more natural starting point is the electron star background and perturb away from there. Realizing then that the fermion equation of motion already depends directly on the dialing parameter $q_{\text {eff }}$ the obvious observables are the single fermion spectral functions in the electron star background. Since one must specify a value for $q_{\text {eff }}$ to compute these, they directly probe the microscopic charge of the fermion and are thus always beyond the strict electron star limit $q \rightarrow 0$. In the next two sections we will compute these and show that they indeed reflect the interpretation of $q_{\text {eff }}$ as the interpolating parameter between the electron star and Dirac Hair BH.

\section{FERMION SPECTRAL FUNCTIONS IN THE ELECTRON STAR BACK- GROUND}

To compute the fermion spectral functions in the electron star background we shall choose a specific representative of the family of electron stars parametrized by $\hat{\beta}$ and $\hat{m}$. Rather than using $\hat{\beta}$ and $\hat{m}$ the metric of an electron star is more conveniently characterized by its Lifshitz-scaling behavior near the interior horizon $r \rightarrow 0$. From the field equations 2.20 the limiting interior behavior of $f(r), g(r), h(r)$ is

$$
f(r)=r^{2 z}+\ldots, \quad g(r)=\frac{g_{\infty}}{r^{2}}+\ldots, \quad h(r)=h_{\infty} r^{z}+\ldots
$$


The scaling behavior is determined by the dynamical critical exponent $z$, which is a function of $\hat{\beta}, \hat{m}$ [7] and it is conventionally used to classify the metric instead of $\hat{\beta}$. The full electron star metric is then generated from this horizon scaling behavior by integrating up an irrelevant RG-flow [19, 20]

$$
f=r^{2 z}\left(1+f_{1} r^{-\alpha}+\ldots\right), \quad g=\frac{g_{\infty}}{r^{2}}\left(1+g_{1} r^{-\alpha}+\ldots\right), \quad h=h_{\infty} r^{z}\left(1+h_{1} r^{-\alpha}+\ldots\right)
$$

with

$$
\alpha=\frac{2+z}{2}-\frac{\sqrt{9 z^{3}-21 z^{2}+40 z-28-\hat{m}^{2} z(4-3 z)^{2}}}{2 \sqrt{\left(1-\hat{m}^{2}\right) z-1}} .
$$

Scaling $f_{1} \rightarrow b f_{1}$ is equal to a coordinate transformation $r \rightarrow b^{1 / \alpha} r$ and $t \rightarrow b^{z / \alpha} t$, and the sign of $f_{1}$ is fixed to be negative in order to be able to match onto an asymptotically $\mathrm{AdS}_{4}$ solution. Thus $f_{1}=-1$ and $g_{1}$ and $h_{1}$ are then uniquely determined by the equations of motion.

Famously, integrating the equations of motion up the RG-flow outwards towards the boundary fails at a finite distance $r_{s}$. This is the edge of the electron star. Beyond the edge of the electron star, there is no fluid present and the spacetime is that of an $\mathrm{AdS}_{4}$ - $\mathrm{RN}$ black hole with the metric

$$
f=c^{2} r^{2}-\frac{\hat{M}}{r}+\frac{\hat{Q}^{2}}{2 r^{2}}, \quad g=\frac{c^{2}}{f}, \quad h=\hat{\mu}-\frac{\hat{Q}}{r} .
$$

Demanding the full metric is smooth at the radius of electron star $r_{s}$ determines the constants $c, \hat{M}$ and $\hat{Q}$. The dual field theory is defined on the plane $d s^{2}=-c^{2} d t^{2}+d x^{2}+d y^{2}$.

The specific electron star background we shall choose without loss of generality is the one with $z=2, \hat{m}=0.36$ (Fig. 1$)^{5}$, smoothly matched at $r_{s} \simeq 4.25252$ onto a AdS-RN black-hole.

The CFT fermion spectral functions now follow from solving the Dirac equation in this background [1, 2]

$$
\left[e_{A}^{\mu} \Gamma^{A}\left(\partial_{\mu}+\frac{1}{4} \omega_{\mu A B} \Gamma^{A B}-i q_{\mathrm{eff}} A_{\mu}\right)-m_{\mathrm{eff}}\right] \Psi=0
$$

\footnotetext{
${ }^{5}$ This background has $c \simeq 1.021, \hat{M} \simeq 3.601, \hat{Q} \simeq 2.534, \hat{\mu} \simeq 2.132, \hat{\beta} \simeq 19.951, g_{\infty} \simeq 1.887, h_{\infty}=$ $1 / \sqrt{2}, \alpha \simeq-1.626, f_{1}=-1, g_{1} \simeq-0.4457, h_{1} \simeq-0.6445$.
} 


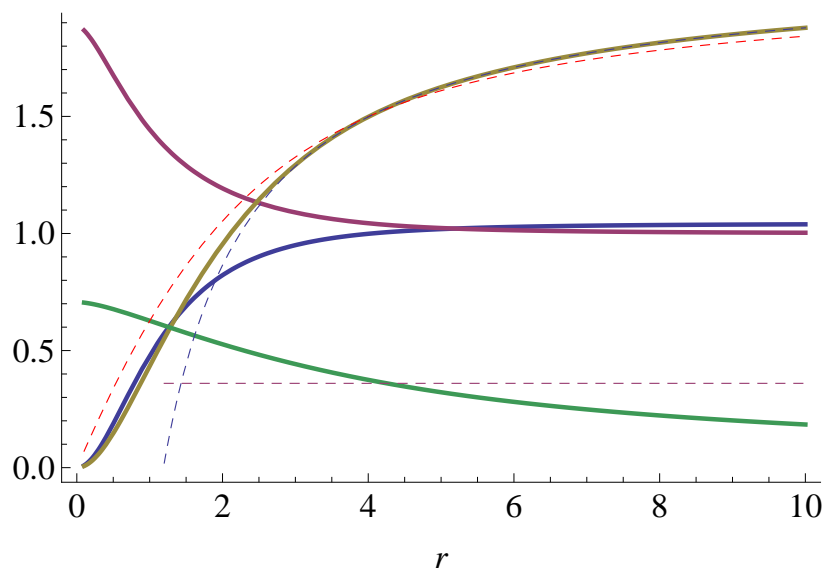

FIG. 1: Electron star metric for $z=2, \hat{m}=0.36, c \simeq 1.021, \hat{M} \simeq 3.601, \hat{Q} \simeq 2.534, \hat{\mu} \simeq 2.132$ compared to pure AdS. Shown are $f(r) / r^{2}$ (Blue), $r^{2} g(r)$ (Red) and $h(r)$ (Orange). The asymptotic AdS-RN value of $h(r)$ is the dashed blue line. For future use we have also given $\mu_{\text {loc }}=h / \sqrt{f}$ (Green) and $\mu_{q_{\text {eff }}}=\sqrt{g^{i i}} h / \sqrt{f}$ (Red Dashed) At the edge of the star $r_{s} \simeq 4.253$ (the intersection of the purple dashed line setting the value of $m_{\text {eff }}$ with $\mu_{\text {loc }}$ ) one sees the convergence to pure AdS in the constant asymptotes of $f(r) / r^{2}$ and $r^{2} g(r)$.

where $q_{\text {eff }}$ and $m_{\text {eff }}$ in terms of the parameters of the electron star equal

$$
q_{\mathrm{eff}}=\left(\frac{\pi^{2} L^{2} \hat{\beta}}{\kappa^{2}}\right)^{1 / 4}, \quad m_{\mathrm{eff}}=q_{\mathrm{eff}} \hat{m}=\hat{m}\left(\frac{\pi^{2} L^{2} \hat{\beta}}{\kappa^{2}}\right)^{1 / 4} .
$$

For a given electron star background, i.e. a fixed $\hat{\beta}, \hat{m}$ the fermion spectral function will therefore depend on the ratio $L / \kappa$. For $L / \kappa \gg \hat{\beta}^{1 / 2}$ the poles in these spectral functions characterize the occupied states in a many-body gravitational Fermi system that is well approximated by the electron star. As $L / \kappa$ is lowered for fixed $\hat{\beta}$ the electron star background becomes a poorer and poorer approximation to the true state and we should see this reflected in both the number of poles in the spectral function and their location.

Projecting the Dirac equation onto two-component $\Gamma^{\underline{r}}$ eigenspinors

$$
\Psi_{ \pm}=\left(-g g^{r r}\right)^{-\frac{1}{4}} e^{-i \omega t+i k_{i} x^{i}}\left(\begin{array}{c}
y_{ \pm} \\
z_{ \pm}
\end{array}\right)
$$

and using isotropy to set $k_{y}=0$, one can choose a basis of Dirac matrices where one obtains two decoupled sets of two simple coupled equations [1]

$$
\begin{aligned}
& \sqrt{g_{i i} g^{r r}}\left(\partial_{r} \mp m_{\mathrm{eff}} \sqrt{g_{r r}}\right) y_{ \pm}=\mp i\left(k_{x}-u\right) z_{\mp} \\
& \sqrt{g_{i i} g^{r r}}\left(\partial_{r} \pm m_{\mathrm{eff}} \sqrt{g_{r r}}\right) z_{\mp}= \pm i\left(k_{x}+u\right) y_{ \pm}
\end{aligned}
$$


where $u=\sqrt{\frac{g_{i i}}{-g_{t t}}}\left(\omega+q_{\mathrm{eff}} h\right)$. In this basis of Dirac matrices the CFT Green's function $G=\left\langle\overline{\mathcal{O}}_{\psi_{+}} i \gamma^{0} \mathcal{O}_{\psi_{+}}\right\rangle$equals

$$
G=\left.\lim _{\epsilon \rightarrow 0} \epsilon^{-2 m L}\left(\begin{array}{cc}
\xi_{+} & 0 \\
0 & \xi_{-}
\end{array}\right)\right|_{r=\frac{1}{\epsilon}}, \quad \text { where } \xi_{+}=\frac{i y_{-}}{z_{+}}, \quad \xi_{-}=-\frac{i z_{-}}{y_{+}}
$$

Rather than solving the coupled equations $(3.8)$ it is convenient to solve for $\xi_{ \pm}$directly [1],

$$
\sqrt{\frac{g_{i i}}{g_{r r}}} \partial_{r} \xi_{ \pm}=-2 m_{\mathrm{eff}} \sqrt{g_{i i}} \xi_{ \pm} \mp\left(k_{x} \mp u\right) \pm\left(k_{x} \pm u\right) \xi_{ \pm}^{2}
$$

For the spectral function $A=\operatorname{Im} \operatorname{Tr} G_{R}$ we are interested in the retarded Green function. This is obtained by imposing in-falling boundary conditions near the horizon $r=0$. Since the electron star is a "zero-temperature" solution this requires a more careful analysis than for a generic horizon. To ensure that the numerical integration we shall perform to obtain the full spectral function has the right infalling boundary conditions, we first solve eq. (3.11) to first subleading order around $r=0$. There are two distinct branches. When $\omega \neq 0$ and $k_{x} r / \omega, r^{2} / \omega$ is small, the in-falling boundary condition near the horizon $r=0$ is (for $z=2$ )

$$
\begin{aligned}
& \xi_{+}(r)=i-i \frac{k_{x} r}{\omega}+i \frac{\left(k_{x}^{2}-2 i m_{\mathrm{eff}} \omega\right) r^{2}}{2 \omega^{2}}-i \frac{f_{1} k_{x} r^{1-\alpha}}{2 \omega}+\ldots \\
& \xi_{-}(r)=i+i \frac{k_{x} r}{\omega}+i \frac{\left(k_{x}^{2}-2 i m_{\mathrm{eff}} \omega\right) r^{2}}{2 \omega^{2}}+i \frac{f_{1} k_{x} r^{1-\alpha}}{2 \omega}+\ldots
\end{aligned}
$$

When $\omega=0$, i.e. $k_{x} r / \omega$ is large, and $r / k_{x} \rightarrow 0$,

$$
\begin{aligned}
& \xi_{+}(r)=-1+\frac{\left(q_{\mathrm{eff}} h_{\infty}+m_{\mathrm{eff}}\right) r}{k_{x}}+\left(\frac{\omega}{k_{x} r}-\frac{\omega}{2 \sqrt{g_{\infty}} k_{x}^{2}}\right)+\ldots \\
& \xi_{-}(r)=1+\frac{\left(q_{\mathrm{eff}} h_{\infty}-m_{\mathrm{eff}}\right) r}{k_{x}}+\left(\frac{\omega}{k_{x} r}-\frac{\omega}{2 \sqrt{g_{\infty}} k_{x}^{2}}\right)+\ldots,
\end{aligned}
$$

the boundary conditions 3.13 become real. As 3.11 are real equations, the spectral function vanishes in this case. This is essentially the statement that all poles in the Green's function occur at $\omega=0$ [17]. Note that the fact that the electron star $\omega=0$ boundary conditions (3.11) are real for all values of $k$ is qualitatively different from the AdS-RN $\omega=0$ boundary conditions (eq. (26) in [1]). In the AdS-RN "quantum-critical" infrared governed by the near horizon $\mathrm{AdS}_{2} \times \mathbb{R}_{2}$ geometry, in general there is a special scale $k_{o}$ below which the boundary condition turns complex. This scale $k_{o}$ is related to the surprising existence of an oscillatory region in the spectral function. One therefore infers that in a scale-ful Lifshitz infrared this oscillatory region is no longer present [13, 21]. We will confirm this in section IV. 


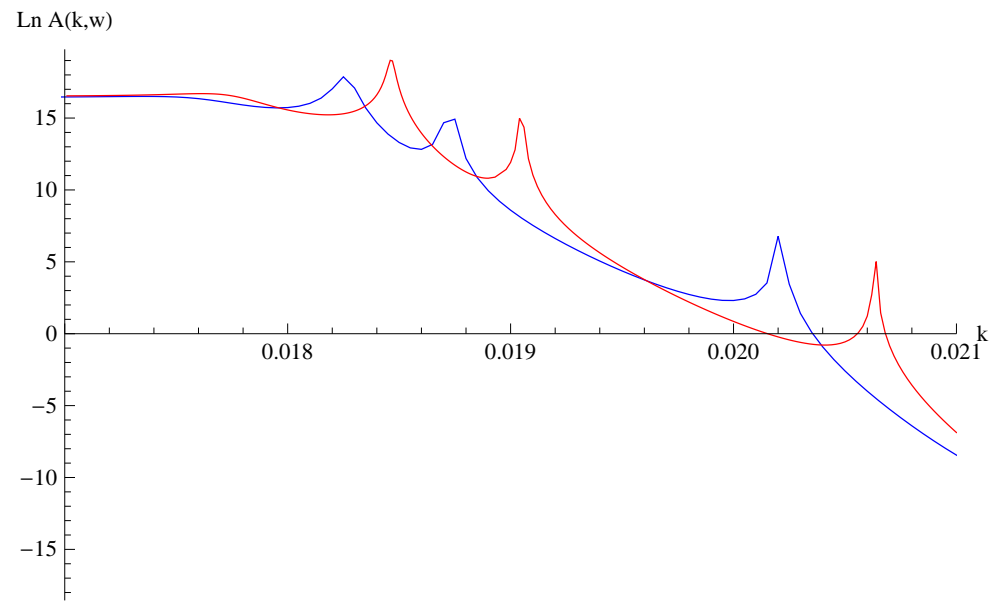

FIG. 2: Electron star MDF spectral functions with multiple peaks as a function of $k$ for $\omega=$ $10^{-5}, z=2, \hat{m}=0.36$. The blue curve is for $\kappa=0.091$; the red curve is for $\kappa=0.090$. Note that the vertical axis is logarithmic. Visible is the rapidly decreasing spectral weight and increasingly narrower width for each successive peak as $k_{F}$ increases.

\section{A. Numerical results and discussion}

We can now solve for the spectral functions numerically. In Fig. 3 we plot the momentumdistribution-function (MDF) (the spectral function as a function of $k$ ) for fixed $\omega=10^{-5}, z=$ $2, \hat{m}=0.36$ while changing the value of $\kappa$. Before we comment on the dependence on $q_{\text {eff }} \sim \kappa^{-1 / 2}$ which studies the deviation away from the adiabatic limit of a given electron star background (i.e. fixed dimensionless charge and fixed dimensionless energy density), there are several striking features that are immediately apparent:

- As expected, there is a multitude of Fermi surfaces. They have very narrow width and their spectral weight decreases rapidly for each higher Fermi-momentum $k_{F}$ (Fig. 27. This agrees with the exponential width $\Gamma \sim \exp \left(-\left(\frac{k^{z}}{\omega}\right)^{1 /(z-1)}\right)$ predicted by [22] for gravitational backgrounds that are Lifshitz in the deep interior, which is the case for the electron star. This prediction is confirmed in [12, 13, 18] and the latter two articles also show that the weight decreases in a corresponding exponential fashion. This exponential reduction of both the width and the weight as $k_{F}$ increases explains why we only see a finite number of peaks, though we expect a very large number. In the next section we will be able to count the number of peaks, even though we cannot resolve them all numerically. 
- The generic value of $k_{F}$ of the peaks with visible spectral weight is much smaller than the effective chemical potential $\mu$ in the boundary field theory. This is quite different from the RN-AdS case where the Fermi momentum and chemical potential are of the same order. A numerical study cannot answer this, but the recent article [13] explains this. ${ }^{6}$

- Consistent with the boundary value analysis, there is no evidence of an oscillatory region.

The most relevant property of the spectral functions for our question is that as $\kappa$ is increased the peak location $k_{F}$ decreases orderly and peaks disappear at various threshold values of $k$. This is the support for our argument that changing $\kappa$ changes the number of microscopic constituents in the electron star. Comparing the the behavior of the various Fermi momenta $k_{F}$ in the electron star with the results in the extremal AdS-RN black-hole, they are qualitatively identical when one equates $\kappa^{-1 / 2} \sim q_{\text {eff }}$ with the charge of the probe fermion. We may therefore infer from our detailed understanding of the behavior of $k_{F}$ for AdS-RN that also for the electron star as $k_{F}$ is lowered peaks truly disappear from the spectrum until by extrapolation ultimately one remains: this is the AdS Dirac hair solution [4].

We can only make this inference qualitatively as the rapid decrease in spectral weight of each successive peak prevents an exact counting of Fermi surfaces in the numerical results for the electron star spectral functions. One aspect that we can already see is that as $\kappa$ decreases all present peaks shift to higher $k$, while new peaks emerging from the left for smaller kappa. This suggests a fermionic version of the UV/IR correspondence where the peak with lowest $k_{F}$ corresponds to the last occupied level, i.e. highest "energy" in the AdS electron star. We will now address both of these points in more detail.

\section{FERMI SURFACE ORDERING: $k_{F}$ FROM A SCHRÖDINGER FORMULA- TION}

Our analysis of the behavior of boundary spectral functions as a function of $\kappa$ relies on the numerically quite evident peaks. Stricly speaking, however, we have not shown that there is

\footnotetext{
${ }^{6}$ In view of the verification of the Luttinger count for electron star spectra in [12, 13, this had to be so.
} 

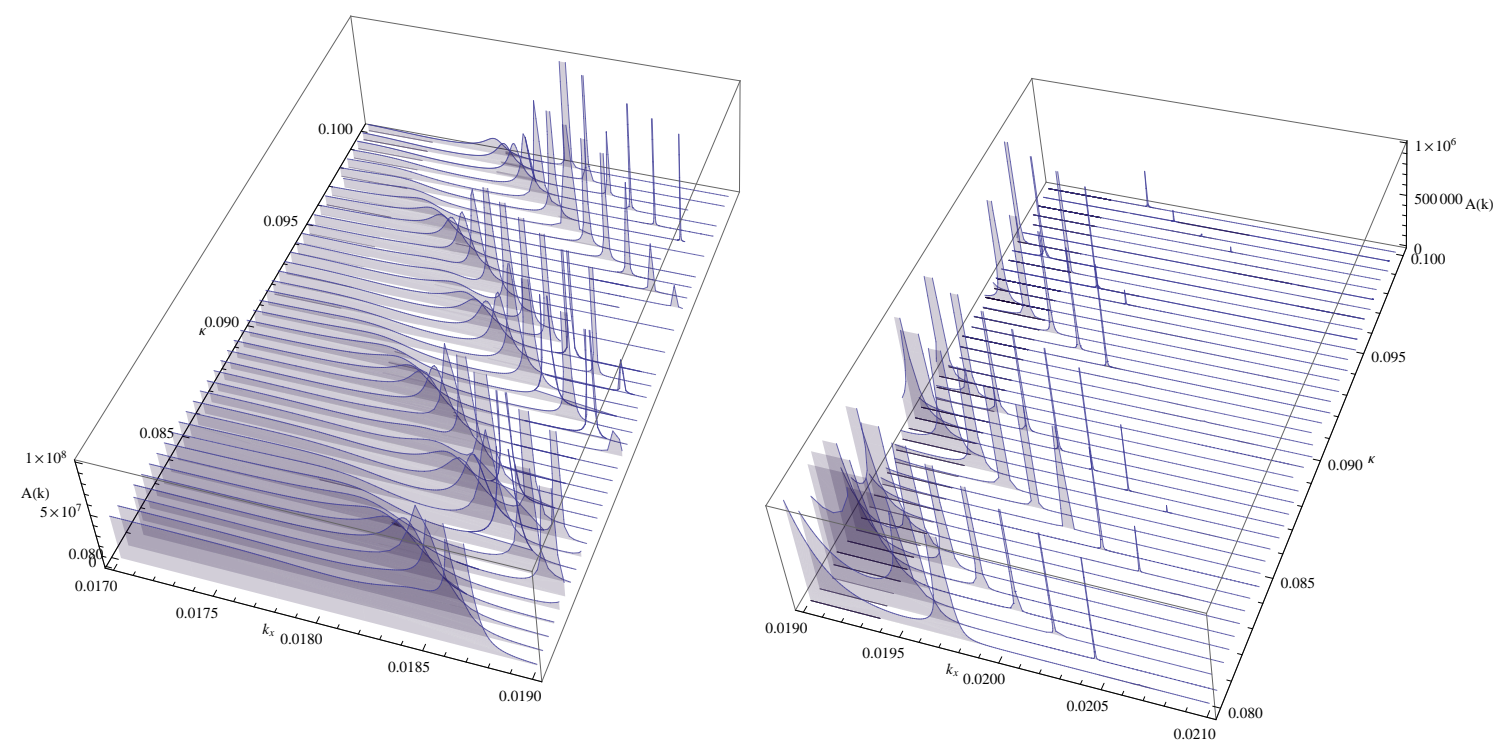

(A)
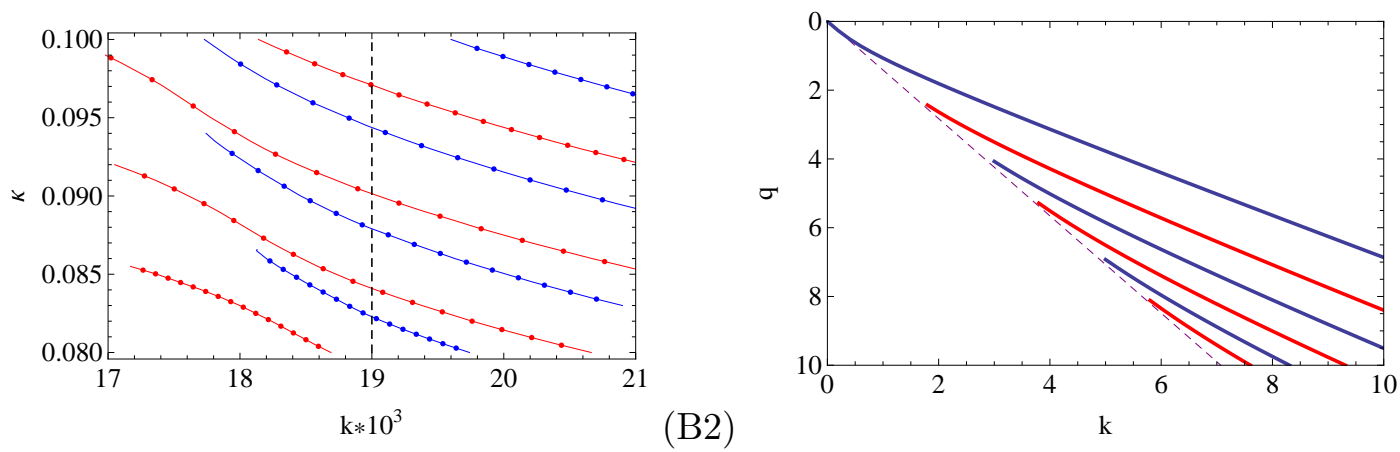

(B1)

FIG. 3: (A) Electron-star MDF spectral functions as a function of $\kappa$ for $z=2, \hat{m}=0.36, \omega=$ $10^{-5}$. Because the peak height and weights decrease exponentially, we present the adjacent ranges $k \in[0.017,0.019]$ and $k \in[0.019,0.021]$ in two different plots with different vertical scale. (B1/B2) Locations of peaks of spectral functions as a function of $\kappa$ : comparison between the electron star (B1) for $z=2, \hat{m}=0.36, \omega=10^{-5}$ (the dashed gray line denotes the artificial separation in the $3 \mathrm{D}$ representations in (A)) and AdS-RN (B2) for $m=0$ as a function of $q$ in units where $\mu=\sqrt{3}$ These two Fermi-surface 'spectra' are qualitatively similar.

a true singularity in the Green's function at $\omega=0, k=k_{F}$. We will do so by showing that the AdS Dirac equation, when recast as a Schrödinger problem has quasi-normalizable solutions at $\omega=0$ for various $k$. As is well known, in AdS/CFT each such solution corresponds to a true pole in the boundary Green's function. Using a WKB approximation for this 
Schrödinger problem we will in addition be able to estimate the number of poles for a fixed $\kappa$ and thereby provide a quantitative value for the deviation from the adiabatic background.

We wish to emphasize that the analysis here is general and captures the behavior of spectral functions in all spherically symmetric and static backgrounds backgrounds alike, whether AdS-RN, Dirac hair or electron star.

The $\omega=0$ Dirac equation 3.5 for one set of components $3.8,3.9$ with the replacement $i y_{-} \rightarrow y_{-}$, equals

$$
\begin{aligned}
& \sqrt{g_{i i} g^{r r}} \partial_{r} y_{-}+m_{\mathrm{eff}} \sqrt{g_{i i}} y_{-}=-\left(k-\hat{\mu}_{q_{\mathrm{eff}}}\right) z_{+}, \\
& \sqrt{g_{i i} g^{r r}} \partial_{r} z_{+}-m_{\mathrm{eff}} \sqrt{g_{i i}} z_{+}=-\left(k+\hat{\mu}_{q_{\mathrm{eff}}}\right) y_{-},
\end{aligned}
$$

where $\hat{\mu}_{q_{\mathrm{eff}}}=\sqrt{\frac{g_{i i}}{-g_{t t}}} q_{\mathrm{eff}} A_{t}$ and we will drop the subscript $x$ on $k_{x}$. In our conventions $z_{+}$ (and $y_{+}$) is the fundamental component dual to the source of the fermionic operator in the CFT [1, 2]. Rewriting the coupled first order Dirac equations as a single second order equation for $z_{+}$:

$$
\begin{aligned}
& \partial_{r}^{2} z_{+}+\mathcal{P} \partial_{r} z_{+}+\mathcal{Q} z_{+}=0, \\
& \mathcal{P}=\frac{\partial_{r}\left(g_{i i} g^{r r}\right)}{2 g_{i i} g^{r r}}-\frac{\partial_{r} \hat{\mu}_{q_{\mathrm{eff}}}}{k+\hat{\mu}_{q_{\mathrm{eff}}}}, \\
& \mathcal{Q}=-\frac{m_{\mathrm{eff}} \partial_{r} \sqrt{g_{i i}}}{\sqrt{g_{i i} g^{r r}}}+\frac{m_{\mathrm{eff}} \sqrt{g_{r r}} \partial_{r} \hat{\mu}_{q_{\mathrm{eff}}}}{k+\hat{\mu}_{q_{\mathrm{eff}}}}-m_{\mathrm{eff}}^{2} g_{r r}-\frac{k^{2}-\hat{\mu}_{q_{\mathrm{eff}}}^{2}}{g_{i i} g^{r r}} .
\end{aligned}
$$

the first thing one notes is that both $\mathcal{P}$ and $\mathcal{Q}$ diverge at some $r=r_{*}$ where $\hat{\mu}_{q_{\text {eff }}}+k=0$. Since $\hat{\mu}_{q_{\text {eff }}}$ is (chosen to be) a positive semidefinite function which increases from $\hat{\mu}_{q_{\text {eff }}}=0$ at the horizon, this implies that for negative $\mathrm{k}$ (with $-k<\left.\hat{\mu}_{q_{\mathrm{eff}}}\right|_{\infty}$ ) the wavefunction is qualitatively different from the wavefunction with positive $k$ which experiences no singularity.The analysis is straightforward if we transform the first derivative away and recast it in the form of a Schrödinger equation by redefining the radial coordinate:

$$
\frac{d s}{d r}=\exp \left(-\int^{r} d r^{\prime} \mathcal{P}\right) \Rightarrow s=c_{0} \int_{r_{\infty}}^{r} d r^{\prime} \frac{\left|k+\hat{\mu}_{\text {eff }}\right|}{\sqrt{g_{i i} g^{r r}}}
$$

where $c_{0}$ is an integration constant whose natural scale is of order $c_{0} \sim q_{\text {eff }}^{-1}$. This is a simpler version of the generalized $k$-dependent tortoise coordinate introduced in [3]. In the new coordinates the equation 4.2 is of the standard form:

$$
\partial_{s}^{2} z_{+}-V(s) z_{+}=0
$$


with potential

$$
V(s)=-\frac{g_{i i} g^{r r}}{c_{0}^{2} \mid k+\hat{\mu}_{\left.q_{\mathrm{eff}}\right|^{2}}} \mathcal{Q} .
$$

The above potential 4.5 can also be written as

$$
V(s)=\frac{1}{c_{0}^{2}\left(k+\hat{\mu}_{\left.q_{\text {eff }}\right)^{2}}\right.}\left[\left(k^{2}+m_{\text {eff }}^{2} g_{i i}-\hat{\mu}_{q_{\text {eff }}}^{2}\right)+m_{\text {eff }} g_{i i} \sqrt{g^{r r}} \partial_{r} \ln \frac{\sqrt{g_{i i}}}{k+\hat{\mu}_{q_{\text {eff }}}}\right] .
$$

We note again the potential singularity for negative $k$, but before we discuss this we first need the boundary conditions. The universal boundary behavior is at spatial infinity and follows from the asymptotic AdS geometry. In the adapted coordinates $r \rightarrow \infty$ corresponds to $s \rightarrow 0$ as follows from $d s / d r \simeq c_{0}\left(k+\left.\hat{\mu}_{q_{\mathrm{eff}}}\right|_{\infty}\right) / r^{2}$. The potential therefore equals

$$
V(s) \simeq \frac{1}{s^{2}}\left(m_{\mathrm{eff}}+m_{\mathrm{eff}}^{2}\right)+\ldots
$$

and the asymptotic behavior of the two independent solutions equals $z_{+}=a_{1} s^{-m_{\text {eff }}}+$ $b_{1} s^{1+m_{\text {eff }}}+\ldots$ The second solution is normalizable and we thus demand $a_{1}=0$.

In the interior, the near-horizon geometry generically is Lifshitz

$$
d s^{2}=-r^{2 z} d t^{2}+\frac{1}{r^{2}} d r^{2}+r^{2}\left(d x^{2}+d y^{2}\right)+\ldots, \quad A=h_{\infty} r^{z} d t+\ldots,
$$

with finite dynamical critical exponent $z$ - AdS-RN, which can be viewed as a special case where $z \rightarrow \infty$, will be given separately. In adapted coordinates the interior $r \rightarrow 0$ corresponds to $s \rightarrow-\infty$ and it is easy to show that in this limit potential behaves as

$$
V(s) \simeq \frac{1}{c_{0}^{2}}+\frac{1}{s^{2}}\left(m_{\mathrm{eff}} \sqrt{g_{\infty}}+m_{\mathrm{eff}}^{2} g_{\infty}-h_{\infty}^{2} q_{\mathrm{eff}}^{2} g_{\infty}\right)+\ldots
$$

Near the horizon the two independent solutions for the wavefunction $z_{+}$therefore behave as

$$
z_{+} \rightarrow a_{0} e^{-s / c_{0}}+b_{0} e^{s / c_{0}}
$$

The decaying solution $a_{0}=0$ is the normalizable solution we seek.

Let us now address the possible singular behavior for $k<0$. To understand what happens, let us first analyze the potential qualitatively for positive $k$. Since the potential is positive semi-definite at the horizon and the boundary, the Schrödinger system (4.4) only has a zero-energy normalizable solution if $V(s)$ has a range $s_{1}<s<s_{2}$ where it is negative. This can only at locations where $k^{2}<\hat{\mu}_{q_{\text {eff }}}^{2}-m_{\text {eff }}^{2} g_{i i}-m_{\text {eff }} g_{i i} \sqrt{g^{r r}} \partial_{r} \ln \frac{\sqrt{g_{i i}}}{k+\hat{\mu}_{q_{\text {eff }}}}$. Defining 
a "renormalized" position dependent mass $m_{\text {ren }}^{2}=m_{\text {eff }}^{2} g_{i i}+m_{\text {eff }} g_{i i} \sqrt{g^{r r}} \partial_{r} \ln \frac{\sqrt{g_{i i}}}{k+\hat{\mu}_{q_{\mathrm{eff}}}}$ this is the intuitive statement that the momenta must be smaller than the local chemical potential $k^{2}<\hat{\mu}_{q_{\mathrm{eff}}}^{2}-m_{\mathrm{ren}}^{2}$. For positive $k$ the saturation of this bound $k^{2}=\hat{\mu}_{q_{\mathrm{eff}}}^{2}-m_{\mathrm{ren}}^{2}$ has at most two solutions, which are regular zeroes of the potential. This follows from the fact that $\hat{\mu}_{q_{\text {eff }}}^{2}$ decreases from the boundary towards the interior. If the magnitude $|k|$ is too large the inequality cannot be satisfied, the potential is strictly positive and no solution exists. For negative $k$, however, the potential has in addition a triple pole at $k^{2}=\hat{\mu}_{q_{\text {eff }}}^{2}$; two poles arise from the prefactor and the third from the $m_{\text {eff }} \partial_{r} \ln \left(k+\hat{\mu}_{q_{\text {eff }}}\right)$ term. This pole always occurs closer to the horizon than the zeroes and the potential therefore qualitatively looks like that in Fig. 4 (Since $\hat{\mu}_{q_{\text {eff }}}$ decreases as we move inward from the boundary, starting with $\hat{\mu}_{q_{\mathrm{eff}}}^{2}>\hat{\mu}_{q_{\mathrm{eff}}}^{2}-\mu^{2}>k^{2}$, one first saturates the inequality that gives the zero in the potential as one moves inward.) Such a potential cannot support a zero-energy bound state, i.e. eq. (4.4) has no solution for negative $k$. In the case $m_{\text {eff }}=0$ a double zero changes the triple pole to a single pole and the argument still holds. This does not mean that there are no $k<0$ poles in the CFT spectral function. They arise from the other physical polarization $y_{+}$of the bulk fermion $\Psi$. From the second set of decoupled first order equations for the other components of the Dirac equation (after replacing $i z_{-} \rightarrow z_{-}$,)

$$
\begin{aligned}
& \sqrt{g_{i i} g^{r r}} \partial_{r} y_{+}-m_{\mathrm{eff}} \sqrt{g_{i i}} y_{+}=-\left(k-\hat{\mu}_{q_{\mathrm{eff}}}\right) z_{-}, \\
& \sqrt{g_{i i} g^{r r}} \partial_{r} z_{-}+m_{\mathrm{eff}} \sqrt{g_{i i}} z_{-}=-\left(k+\hat{\mu}_{q_{\mathrm{eff}}}\right) y_{+},
\end{aligned}
$$

and the associated second order differential EOM for $y_{+}$:

$$
\begin{aligned}
& \partial_{r}^{2} y_{+}+\mathcal{P} \partial_{r} y_{+}+\mathcal{Q}=0, \\
& \mathcal{P}=\frac{\partial_{r}\left(g_{i i} g^{r r}\right)}{2 g_{i i} g^{r r}}-\frac{\partial_{r} \hat{\mu}_{q_{\text {eff }}}}{-k+\hat{\mu}_{q_{\text {eff }}}}, \\
& \mathcal{Q}=-\frac{m_{\text {eff }} \partial_{r} \sqrt{g_{i i}}}{\sqrt{g_{i i} g^{r r}}}+\frac{m_{\text {eff }} \sqrt{g_{r r}} \partial_{r} \hat{\mu}_{q_{\text {eff }}}}{-k+\hat{\mu}_{q_{\text {eff }}}}-m_{\text {eff }}^{2} g_{r r}-\frac{k^{2}-\hat{\mu}_{q_{\text {eff }}}^{2}}{g_{i i} g^{r r}},
\end{aligned}
$$

one sees that the Schrödinger equation for $y_{+}$is the $k \rightarrow-k$ image of the equation (4.4) for $z_{+}$and thus $y_{+}$will only have zero-energy solutions for $k<0$. For simplicity we will only analyze the $z_{+}$case. Note that this semi-positive definite momentum structure of the poles is a feature of any AdS-to-Lifshitz metric different from AdS-RN, where one can have negative $k$ solutions [3].

The exact solution of 4.4 with the above boundary conditions corresponding to poles in the CFT spectral function is difficult to find. By construction the system is however 

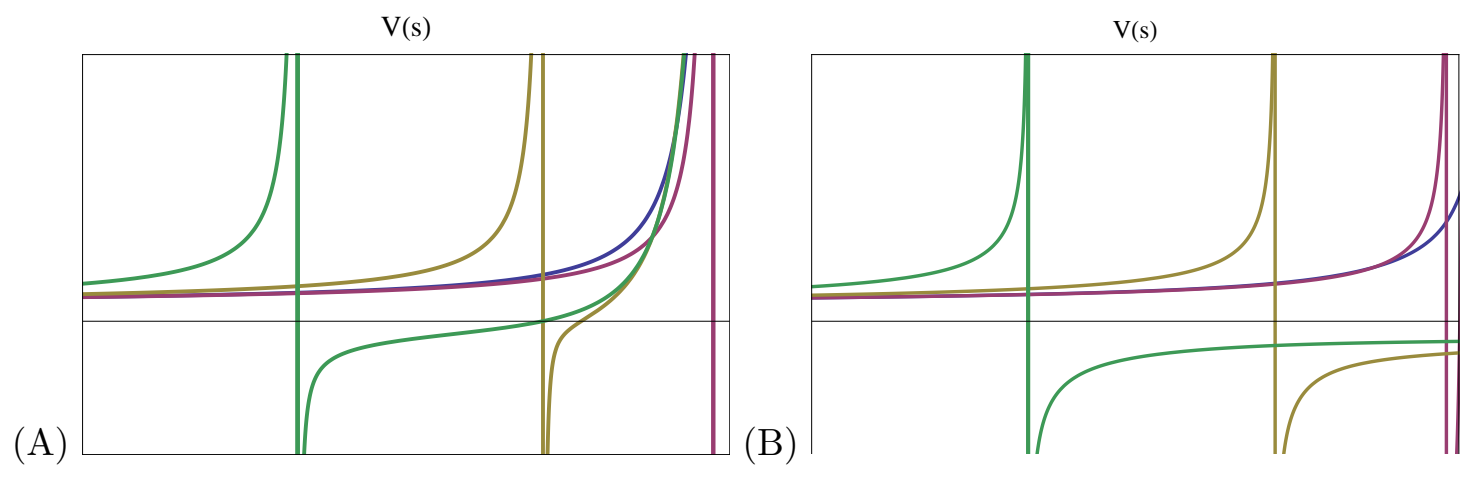

FIG. 4: The behavior of the Schrödinger potential $V(s)$ for $z_{+}$when $k$ is negative. Such a potential has no zero-energy bound state. The potential is rescaled to fit on a finite range. As $|k|$ is lowered below $k_{\max }$ for which the potential is strictly positive, a triple pole appears which moves towards the horizon on the left (Fig A. The Blue,Red,Orange,Green curves are decreasing in $|k|$ ). The pole hits the horizon for $k=0$ and disappears. Fig B. shows the special case $m_{\text {eff }}=0$ where two zeroes collide with two of the triple poles to form a single pole.

equivalent to a Schrödinger problem of finding a zero energy solution $z_{+}$in the potential 4.5 and can be solved in the WKB approximation (see e.g. [3, 23]). The WKB approximation holds when $\left|\partial_{s} V\right| \ll|V|^{3 / 2}$. Notice that this is more general than the background adiabacity limit $m_{\text {eff }} \gg 1, q_{\text {eff }} \gg 1$ with $\hat{\beta}, \hat{m}$ fixed. Combining background adiabaticity with a scaling limit $k \gg 1, m_{\text {eff }} \gg 1, q_{\text {eff }} \gg 1$ with $c_{0} k$ fixed and $k$ is parametrically larger than $\hat{\mu}_{q_{\text {eff }}}$ one recovers the WKB potential solved in [12, 13]. As our aim is to study the the deviation away from the background adiabatic limit we will be more general and study the WKB limit of the potential itself, without direct constraints on $q_{\text {eff }}, m_{\text {eff }}$. And rather than testing the inequality $\left|\partial_{s} V\right| \ll|V|^{3 / 2}$ directly, we will rely on the rule of thumb that the WKB limit is justified when the number of nodes in the wave-function is large. We will therefore estimate the number $n$ of bound states and use $n \gg 1$ as an empirical justification of our approach. ${ }^{7}$ With this criterion we will be able to study the normalizable solutions to the Dirac equation/pole structure of the CFT spectral functions as a function of $\kappa / L$.

The potential is bounded both in the AdS boundary and at the horizon, and decreases towards intermediate values of $r$. We therefore have a standard WKB solution consisting of

\footnotetext{
${ }^{7}$ A large number of bound states $n$ implies $\left|\partial_{s} V\right| \ll|V|^{3 / 2}$ if the potential has a single minimum, but as is well known there are systems, e.g. the harmonic oscillator, where the WKB approximation holds for small $n$ as well.
} 
three regions:

- In the regions where $V>0$, the solution decays exponentially:

$$
z_{+}=c_{1,2} V^{-1 / 4} \exp \left( \pm \int_{r_{1,2}}^{r} d r^{\prime}\left[c_{0} \sqrt{g^{i i} g_{r r}}\left(k+\hat{\mu}_{q_{\text {eff }}}\right) \sqrt{V}\right]\right) .
$$

Here $r_{1}, r_{2}$ are the turning points where $V\left(r_{1}\right)=0=V\left(r_{2}\right)$.

- In the region $r_{1}<r<r_{2}$, i.e. $V<0$, the solution is

$$
z_{+}=c_{3}(-V)^{-1 / 4} \operatorname{Re}\left[\exp \left(i \int_{r_{1}}^{r} d r^{\prime}\left[c_{0} \sqrt{g^{i i} g_{r r}}\left(k+\hat{\mu}_{q_{\mathrm{eff}}}\right) \sqrt{-V}\right]-i \pi / 4\right)\right]
$$

with the constant phase $-i \pi / 4$ originating in the connection formula at the turning point $r_{1}$.

Finding a WKB solution shows us that the peaks seen numerically are true poles in the spectral function. But it also allows us to estimate the number of peaks that the numerical approach could not resolve. The WKB quantization condition

$$
\int_{r_{1}}^{r_{2}} d r^{\prime}\left[c_{0} \sqrt{g^{i i} g_{r r}}\left(k+\hat{\mu}_{q_{\mathrm{eff}}}\right) \sqrt{-V}\right]=\pi(n+1 / 2)
$$

counts the number of bound states with negative semi-definite energy. Note that $n$ does not depend on the integral constant as there is also a factor $1 / c_{0}$ in $\sqrt{-V}$. Since $V$ depends on $k$, we will see that as we increase $k$ this number decreases. The natural interpretation in the context of a bulk many-body Fermi system is that this establishes the ordering of the the filling of all the $\omega=0$ momentum shells in the electron star. For a fixed $k$ one counts the modes that have been previously occupied and, consistent with our earlier deduction, the lowest/highest $k_{F}$ corresponds to the last/first occupied state. Though counterintuitive from a field theory perspective where normally $E \sim k_{F}$, this $\mathrm{UV} / \mathrm{IR}$ correspondence is very natural from the AdS-bulk, if one thinks of the electron star as a trapped electron gas. The last occupied state should then be the outermost state from the center, but this state has the lowest effective chemical potential and hence lowest $k_{F}$.

Let us now show this explicitly by analyzing the potential and the bound states in the electron star and AdS-RN. 


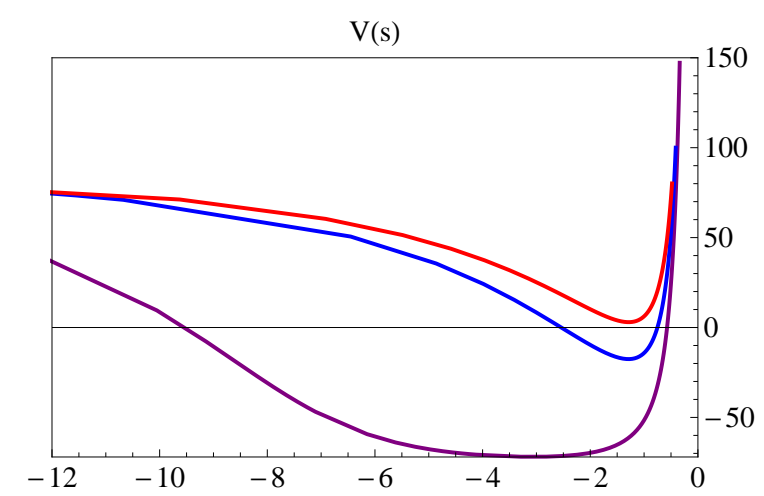

(A)

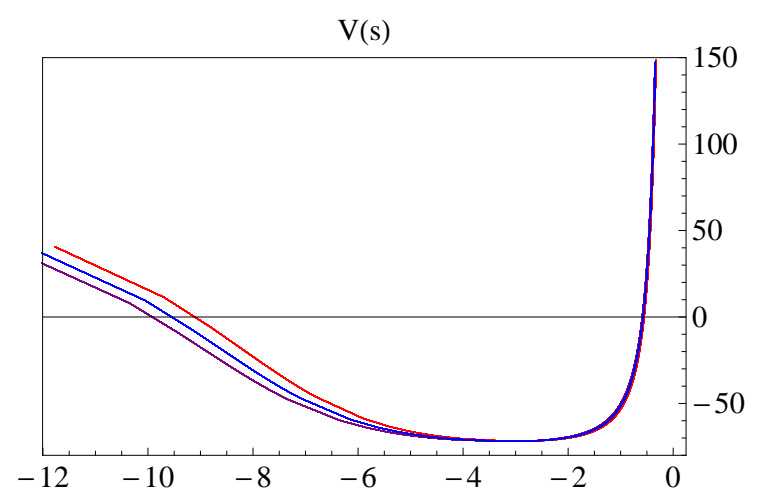

(B)

FIG. 5: The Schrödinger potential $V(s)$ for the fermion component $z_{+}$of in the ES background $\hat{m}=0.36, z=2, c_{0}=0.1$. Fig. A. shows the dependence on the momentum $k=0.0185$ (Purple), $k=5$ (Blue), $k=10$ (Red) for $\kappa=0.092$. Fig. B. shows the dependence on $\kappa=0.086$ (Purple), $\kappa=0.092$ (Blue), $\kappa=0.1$ (Red) for $k=0.0185$. Recall that $s=0$ is the AdS boundary and $s=-\infty$ is the near-horizon region.

\section{Electron star}

The potential (4.6) for the electron star is given in Fig. 5 and the number of bound states as a function of $k$ in Fig. 6. As stated the number of states decreases with increasing $k$, consistent with the analogy of the pole distribution of the spectral functions compared with AdS-RN. Moreover, we clearly see the significant increase in the number of states as we decrease $\kappa / L$ thereby improving the adiabaticity of the background. This vividly illustrates that the adiabatic limit corresponds to a large number of constituents. As all numbers of states are far larger than one, the use of the WKB is justified.

\section{The Reissner-Nordström case}

For AdS-RN the Schrödinger analysis requires a separate discussion of the near horizon boundary conditions, which we present here for completeness and comparison. Part of this 

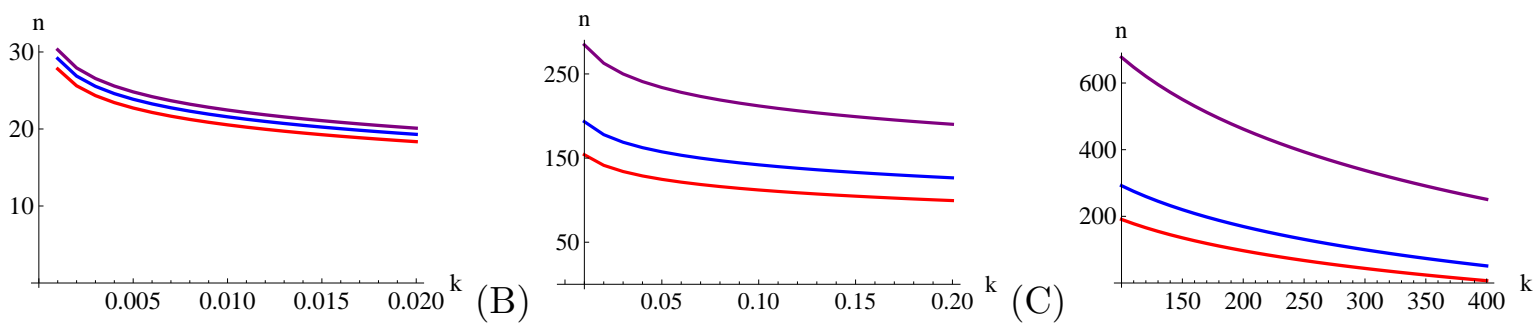

FIG. 6: The WKB estimate of the number of bound states $n$ as a function of the momentum $k$ for $\kappa=0.086$ (Purple), 0.092(Blue), 0.1(Red) (Fig A.); for $\kappa=0.001$ (Purple), 0.002(Blue), 0.003(Red) (Fig B.) and for $\kappa=10^{-5}$ (Purple), $3 \times 10^{-5}$ (Blue), $5 \times 10^{-5}$ (Red) (Fig C.). Note the parametric increase in number of states as the adiabaticity of the background improves for smaller $\kappa$. Both figures are for the electron star background with $\hat{m}=0.36, z=2$. Since $n \gg 1$ in all cases, WKB gives a valid estimate.

analysis is originally worked out in [3]. The AdS-RN black hole with metric

$$
\begin{aligned}
d s^{2} & =L^{2}\left(-f(r) d t^{2}+\frac{d r^{2}}{f(r)}+r^{2}\left(d x^{2}+d y^{2}\right)\right) \\
f(r) & =r^{2}\left(1+\frac{3}{r^{4}}-\frac{4}{r^{3}}\right) \\
A & =\mu\left(1-\frac{1}{r}\right) d t
\end{aligned}
$$

has near horizon geometry $\mathrm{AdS}_{2} \times \mathbb{R}^{2}$

$$
\begin{aligned}
d s^{2} & =-6(r-1)^{2} d t^{2}+\frac{d r^{2}}{6(r-1)^{2}}+\left(d x^{2}+d y^{2}\right) \\
A & =\sqrt{3}(r-1) d t
\end{aligned}
$$

A coordinate redefinition of $r$ in eq. (4.8) to $r=\left(r_{A d S_{2}}-1\right)^{1 / z}$ shows that this corresponds to a dynamical critical exponent $z=\infty$ and is outside the validity of the previous analysis.

Before we proceed, recall that the existence of $\mathrm{AdS}_{2} \times \mathbb{R}^{2}$ near-horizon region allows for a semi-analytic determination of the fermion spectral functions with the self-energy $\Sigma \sim \omega^{2 \nu_{k_{F}}}$ controlled by the IR conformal dimension $\delta_{k}=1 / 2+\nu_{k}$ with

$$
\nu_{k}=\frac{1}{\sqrt{6}} \sqrt{m^{2}+k^{2}-\frac{q^{2}}{2}} .
$$

When $\nu_{k}$ is imaginary, which for $q^{2}>2 m^{2}$ always happens for small $k$, the spectral function exhibits oscillatory behavior, but generically has finite weight at $\omega=0$. When $\nu_{k}$ is real, 


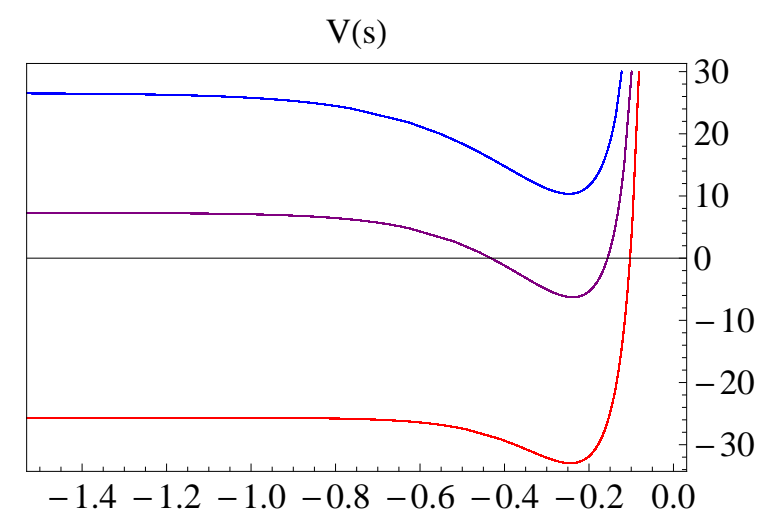

(A)

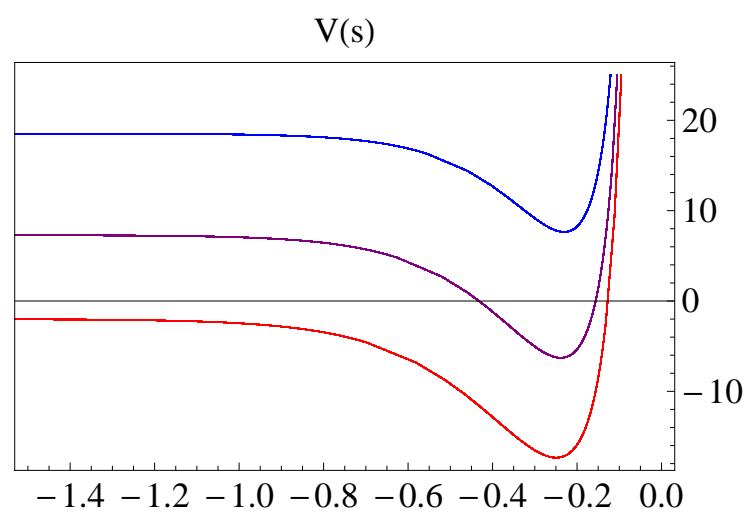

(B)

S

FIG. 7: The Schrödinger potential $V(s)$ for the fermion component $z_{+}$of in the AdS-RN background $r_{+}=1, \mu=\sqrt{3}, g_{F}=1, m L=0.4, c_{0}=0.1$. Fig. A. shows the dependence on the momentum $k=1$ (Red), $k=2$ (Purple), $k=3$ (Blue) for charge $q=2.5$. Fig. B. shows the dependence on the charge $q$ - analogous to $\kappa$ in the ES background - . Shown are the values $q=2$ (Blue), $q=2.5$ (Purple), $q=3$ (Red) for the momentum $k=2$. In both figures the Red potentials correspond to the oscillatory region $\nu_{k}^{2}<0$, the Purple potentials show the generic shape that can support an $\omega=0$ bound state, and the Blue potentials are strictly positive and no zero-energy bound state is present. Recall that $s=0$ is the AdS boundary and $s=-\infty$ is the near-horizon region.

there are poles in the spectral functions at a finite number of different Fermi momenta $k_{F}$. The associated quasiparticles can characterize a non-FL $\left(\nu_{k_{F}}<1 / 2\right)$, a marginal FL $\left(\nu_{k_{F}}=1 / 2\right)$ or irregular FL $\left(\nu_{k_{F}}>1 / 2\right)$ with linear dispersion but width $\Gamma \neq \omega^{2}[3]$.

The analytic form of the near-horizon metric allows us to solve exactly for the near horizon potential $V$ in terms of $s=\frac{c_{0}}{\sqrt{6}}(k+q / \sqrt{2}) \ln (r-1)+\ldots$ As noted in [3] one remarkably obtains that the near-horizon potential for $s \rightarrow-\infty$ is proportional to the self-energy exponent:

$$
V(s) \simeq \frac{6}{c_{0}^{2}(k+q / \sqrt{2})^{2}} \nu_{k}^{2}+\ldots
$$

One immediately recognizes the oscillatory region $\nu_{k}^{2}<0$ of the spectral function as an $\omega=0$ Schrödinger potential which is "free" at the horizon $s=-\infty$ (Fig. 7) and no bound state can form. Comparing with our previous results, we see that this oscillatory region is a distinct property of AdS-RN. For any Lifshitz near-horizon metric the potential is always positive-definite near the horizon and all $\omega=0$ solutions will be bounded. (see also [12, 13]). As we increase $k, \nu_{k}^{2}$ becomes positive, then the AdS-RN potential is also positive at the 


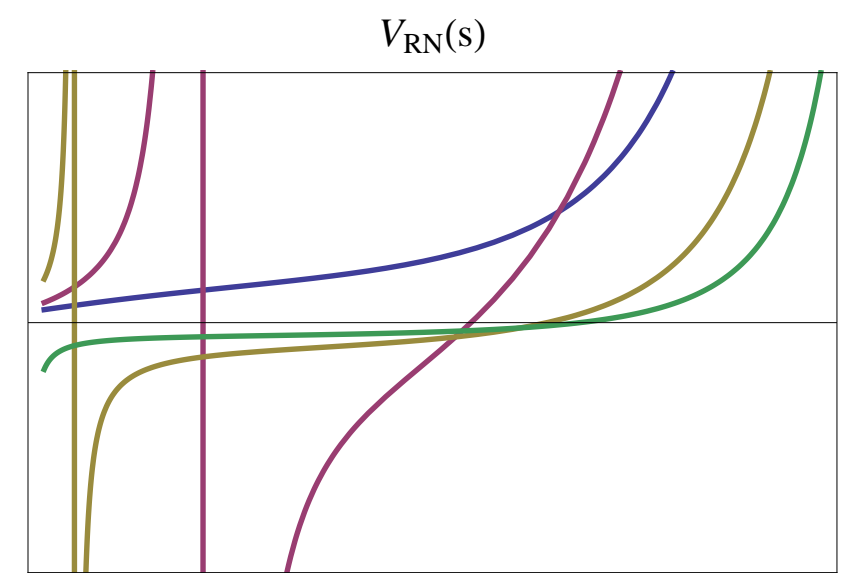

FIG. 8: The qualitative behavior for negative $k$ of the Schrödinger potential $V(s)$ for the fermion component $z_{+}$of in the AdS-RN background $r_{+}=1, \mu=\sqrt{3}, g_{F}=1, m L=0.1$. The radial coordinate has been rescaled to a finite domain such that the full potential can be represented in the figure; on the right is the AdS boundary and left is the near-horizon region and the range is slightly extended beyond the true horizon, which is exactly at the short vertical line-segments on the right. Potentials are given for $q=12 / \sqrt{3}, k=-15$ (Blue) for which the potential is strictly positive, $k=-10$ (Red), $k=-7$ (Orange), which both have triple poles and the pole can be seen to move towards the horizon on the left as $k$ decreases, and $k=-4$ (Green) which has no pole and a finite negative value at the horizon. The pole disappears for $|k|<q / \sqrt{2}$ leaving a regular bounded potential which can support zero-energy bound states.

horizon and bound zero-energy states can form. Increasing $k$ further, one reaches a maximal $k_{\max }$, above which the potential is always positive and no zero-energy bound state exists anymore.

Because the near-horizon boundary conditions for AdS-RN differ from the general analysis, the possible singularity in the potential for $k<0$ also requires a separate study. This is clearly intimately tied to the existence of an oscillatory regime in the spectral function, as the previous analysis does apply for $\nu_{k}^{2}>0$. The clearest way to understand what happens for $\nu_{k}^{2}<0$ is to analyze the potential explicitly. Again if $|k|>k_{\max }$ the potential is strictly positive definite, and no zero-energy bound state exists. As we decrease the magnitude of $k<0$, a triple pole will form near the boundary when $k=-\hat{\mu}_{q_{\text {eff }}}(s)$, soon followed by a zero at $k=-\sqrt{\hat{\mu}_{q_{\text {eff }}}(s)^{2}-m_{\text {ren }}(s)^{2}}$ (see Fig. 4). As we approach the horizon, in the general case where $\lim _{r \rightarrow 0} \hat{\mu}_{q_{\text {eff }}}=h_{\infty} q_{\text {eff }} r+\ldots$, this pole at $r_{*}=-k /\left(h_{\infty} q_{\text {eff }}\right)$ hits the horizon and disap- 
(A)

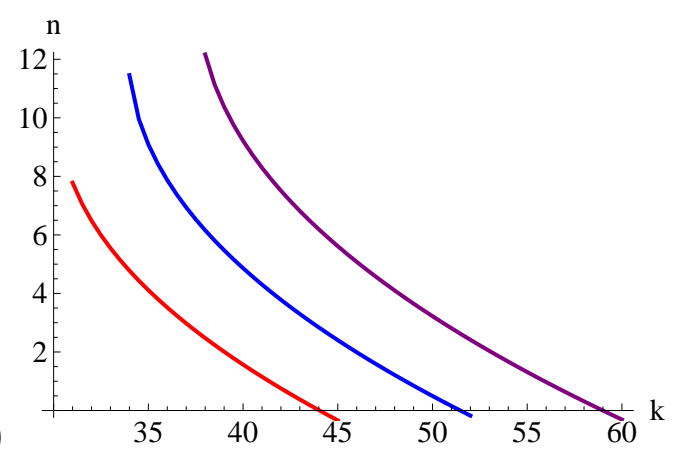

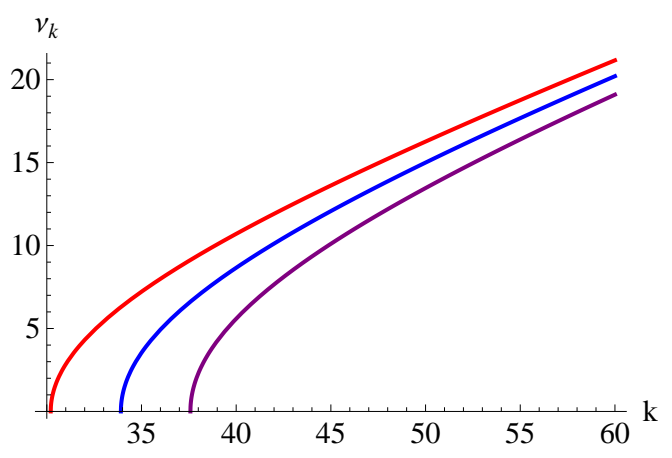

FIG. 9: The WKB estimate of the number of bound states $n$ in the AdS-RN Schrödinger potential for $z_{+}$with $m L=10$. The WKB approximation only applies to large values of the charge $q=45$ (Red), $q=50$ (Blue), $q=55$ (Purple). Fig B. gives the associated values of the IR conformal dimension $\nu_{k}=\frac{1}{\sqrt{6}} \sqrt{m^{2}+k^{2}-\frac{q^{2}}{2}}$. Both figures are for the extremal AdS-RN background with $\mu=\sqrt{3}, r_{+}=1, g_{F}=1$.

pears precisely when $k=0$. In AdS-RN, however, where $\lim _{r \rightarrow 1} \hat{\mu}_{q_{\text {eff }}}=\frac{q}{\sqrt{2}}+\frac{\sqrt{2} q}{3}(r-1)+\ldots$, the pole at $r_{*}^{R N}-1=\frac{3}{\sqrt{2} q}\left(k+\frac{q}{\sqrt{2}}\right)$ hits the horizon and disappears at $k=-\frac{q}{\sqrt{2}}$. For negative values of $k$ whose magnitude is less than $|k|<\frac{q}{\sqrt{2}}$, the potential is regular and bounded and can and does have zero-energy solutions. Fig. IV shows this disappearance of the pole for the AdS-RN potential.

Counting solutions through WKB is also more complicated for AdS-RN. For $\mathcal{O}(1)$ values of $q$ there are only few Fermi surfaces and the WKB approximation does not apply. For large $q$ it does, however. For completeness we show the results in Fig. 9.

\section{CONCLUSION AND DISCUSSION}

These electron star spectral function results answer two of the three questions raised in the introduction directly.

- They show explicitly how the fermion wavefunctions in their own gravitating potential well are ordered despite the fact that they all have strictly vanishing energy: In a fermionic version of the UV-IR correspondence they are ordered inversely in $k$, with the "lowest" / first occupied state having the highest $k$ and the "highest" / last occupied state having the lowest $k$. With the qualitative AdS/CFT understanding that scale corresponds to distance away from the interior, one can intuitively picture this as 
literally filling geometrical shells of the electron star, with the outermost/highest/last shell at large radius corresponding to the wavefunction with lowest local chemical potential and hence lowest $k$.

- The decrease of the number of bound states - the number of occupied wavefunctions in the electron star - as we decrease $q_{\text {eff }}=\hat{\beta}^{1 / 4} \sqrt{\frac{\pi L}{\kappa}}$ for a fixed electron star background extrapolates naturally to a limit where the number of bound states is unity. This extrapolation pushes the solution beyond its adiabatic regime of validity. In principle we know what the correct description in this limit is: it is the AdS Dirac Hair solution constructed in [4. The dependence of the number of bound states on $\kappa / L$ therefore illustrates that the electron star and Dirac Hair solutions are two limiting cases of the gravitationally backreacted Fermi gas.

With this knowledge we can schematically classify the groundstate solutions of AdS EinsteinMaxwell gravity minimally coupled to charged fermions at finite charge density. For large mass $m L$ in units of the constituent charge $q$, the only solution is a charged AdS-ReissnerNördstrom black hole. For a low enough mass-to-charge ratio, the black hole becomes unstable and develops hair. If in addition the total charge density $Q$ is of the order of the microscopic charge $q$ this hairy solution is the Dirac Hair configuration constructed in [4, whereas in the limit of large total charge density $Q$ one can make an adiabatic Thomas Fermi approximation and arrives a la Tolman-Oppenheimer-Volkov at an electron star (Fig. 10 .

Translating this solution space through the AdS/CFT correspondence one reads off that in the dual strongly coupled field theory, one remains in the critical state if the ratio of the scaling dimension to the charge $\Delta / q$ is too large. For a small enough value of this ratio, the critical state is unstable and forms a novel scaleful groundstate. The generic condensed matter expectation of a unique Fermi liquid is realized if the total charge density is of the same order as the constituent charge. Following [12, 13] and [14 16] the state for $Q \gg q$ is some deconfined Fermi liquid.

The gravity description of either limit has some deficiencies, most notably the lack of an electron star wavefunction at infinity and the unnatural restriction to $Q=q$ for the Dirac Hair solution. A generic solution for $Q \geq q$ with wavefunction tails extending to infinity as the Dirac hair would be a more precise holographic dual to the strongly interacting large 

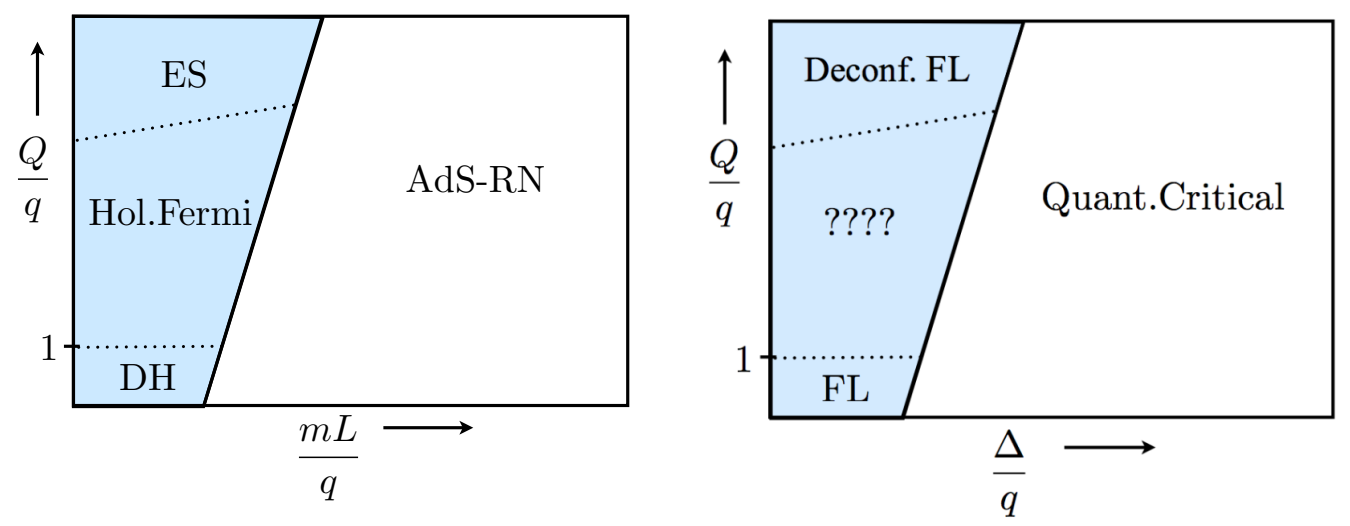

FIG. 10: Schematic diagram of the different groundstate solutions of strongly coupled fermions implied by holography for fixed charge density $Q$. Here $q$ is the constituent charge of the fermions and $m L \sim \Delta$ the mass/conformal scaling dimension of the fermionic operator. One has the gravitational electron star (ES)/Dirac Hair (DH) solution for large/small $Q / q$ and small $m L / q$ dual a deconfined Fermi liquid/regular Fermi liquid in the CFT. For $m L / q \sim \Delta / q$ large the groundstate remains the fermionic quantum critical state dual to AdS-RN.

$N$ Fermi system. Any CFT information can then be cleanly read off at the AdS boundary. A naive construction could be to superpose Dirac Hair onto the electron star; in principle one can achieve this solution by a next order Hartree-Fock or Local Density Approximation computation.

This best-of-both-worlds generic solution ought to be the true holographic dual of the strongly interacting Fermi ground state. If one is able to answer convincingly how this system circumvents the wisdom that the groundstate of an interacting many-body system of fermions is a generic single quasiparticle Landau Fermi liquid, then one would truly have found a finite density Fermi system that does not refer at any stage to an underlying perturbative Fermi gas.

\section{Acknowledgements}

We thank S. Hartnoll, A. Karch, H. Liu, T. K. Ng and B. Overbosch for discussions and correspondence. KS is very grateful to the Hong Kong Institute for Advanced Studies for the hospitality during the completion of this work. This research was supported in part by a 
VIDI Innovative Research Incentive Grant (K. Schalm) from the Netherlands Organisation for Scientific Research (NWO), a Spinoza Award (J. Zaanen) from the Netherlands Organisation for Scientific Research (NWO) and the Dutch Foundation for Fundamental Research on Matter (FOM).

[1] H. Liu, J. McGreevy and D. Vegh, Non-Fermi liquids from holography, arXiv:0903.2477] [hepth].

[2] M. Cubrovic, J. Zaanen and K. Schalm, String Theory, Quantum Phase Transitions and the Emergent Fermi-Liquid, Science 325, 439 (2009) arXiv:0904.1993 [hep-th]].

[3] T. Faulkner, H. Liu, J. McGreevy and D. Vegh, Emergent quantum criticality, Fermi surfaces, and AdS2, arXiv:0907.2694 [hep-th].

[4] M. Cubrovic, J. Zaanen and K. Schalm, Constructing the AdS dual of a Fermi liquid: AdS Black holes with Dirac hair, arXiv:1012.5681 [hep-th].

[5] M. Cubrovic, J. Zaanen and K. Schalm, Fermi Liquid stability at strong coupling from AdS Black holes with Dirac hair, to appear.

[6] S. A. Hartnoll, J. Polchinski, E. Silverstein and D. Tong, JHEP 1004, 120 (2010) arXiv:0912.1061 [hep-th]].

[7] S. A. Hartnoll and A. Tavanfar, Electron stars for holographic metallic criticality, arXiv:1008.2828 [hep-th].

[8] J. de Boer, K. Papadodimas and E. Verlinde, Holographic Neutron Stars, JHEP 1010, 020 (2010) arXiv:0907.2695 [hep-th]].

[9] X. Arsiwalla, J. de Boer, K. Papadodimas and E. Verlinde, Degenerate Stars and Gravitational Collapse in AdS/CFT, arXiv:1010.5784 [hep-th].

[10] V. G. M. Puletti, S. Nowling, L. Thorlacius and T. Zingg, Holographic metals at finite temperature, arXiv:1011.6261 [hep-th].

[11] S. A. Hartnoll and P. Petrov, Electron star birth: A continuous phase transition at nonzero density, arXiv:1011.6469 [hep-th].

[12] S. A. Hartnoll, D. M. Hofman and D. Vegh, Stellar spectroscopy: Fermions and holographic Lifshitz criticality, arXiv:1105.3197 [hep-th].

[13] N. Iqbal, H. Liu and M. Mezei, Semi-local quantum liquids, arXiv:1105.4621 [hep-th]. 
[14] S. A. Hartnoll, D. M. Hofman and A. Tavanfar, Holographically smeared Fermi surface: Quantum oscillations and Luttinger count in electron stars, arXiv:1011.2502 [hep-th].

[15] S. Sachdev, Holographic metals and the fractionalized Fermi liquid, Phys. Rev. Lett. 105, 151602 (2010) arXiv:1006.3794 [hep-th]].

[16] L. Huijse and S. Sachdev, Fermi surfaces and gauge-gravity duality, arXiv:1104.5022 [hep-th].

[17] H. Liu, T. Faulkner, private communication.

[18] N. Iizuka, N. Kundu, P. Narayan and S. P. Trivedi, Holographic Fermi and Non-Fermi Liquids with Transitions in Dilaton Gravity, arXiv:1105.1162 [hep-th].

[19] S. S. Gubser and A. Nellore, Ground states of holographic superconductors, Phys. Rev. D 80, 105007 (2009) arXiv:0908.1972 [hep-th]].

[20] K. Goldstein, S. Kachru, S. Prakash and S. P. Trivedi, Holography of Charged Dilaton Black Holes, JHEP 1008, 078 (2010) [arXiv:0911.3586 [hep-th]].

[21] D. Guarrera and J. McGreevy, Holographic Fermi surfaces and bulk dipole couplings, arXiv:1102.3908 [hep-th].

[22] T. Faulkner and J. Polchinski, Semi-Holographic Fermi Liquids, arXiv:1001.5049 [hep-th].

[23] G. T. Horowitz and M. M. Roberts, Zero Temperature Limit of Holographic Superconductors, JHEP 0911, 015 (2009) arXiv:0908.3677 [hep-th]].

[24] T. Faulkner, N. Iqbal, H. Liu, J. McGreevy and D. Vegh, Holographic non-Fermi liquid fixed points, arXiv:1101.0597 [hep-th]. 